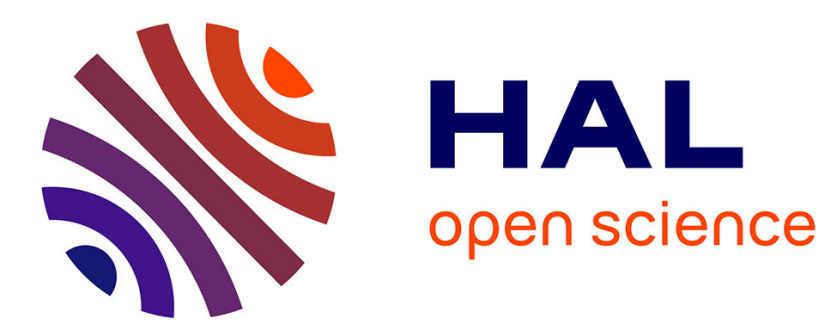

\title{
Testing the parametric form of the volatility in continuous time diffusion models - a stochastic process approach
}

Holger Dette, Mark Podolskij

\section{- To cite this version:}

Holger Dette, Mark Podolskij. Testing the parametric form of the volatility in continuous time diffusion models - a stochastic process approach. Econometrics, 2008, 143 (1), pp.56. 10.1016/j.jeconom.2007.08.002 . hal-00501800

\section{HAL Id: hal-00501800 \\ https://hal.science/hal-00501800}

Submitted on 12 Jul 2010

HAL is a multi-disciplinary open access archive for the deposit and dissemination of scientific research documents, whether they are published or not. The documents may come from teaching and research institutions in France or abroad, or from public or private research centers.
L'archive ouverte pluridisciplinaire HAL, est destinée au dépôt et à la diffusion de documents scientifiques de niveau recherche, publiés ou non, émanant des établissements d'enseignement et de recherche français ou étrangers, des laboratoires publics ou privés. 


\section{Author's Accepted Manuscript}

Testing the parametric form of the volatility in continuous time diffusion models - a stochastic process approach

Holger Dette, Mark Podolskij

PII: S0304-4076(07)00159-5

DOI: doi:10.1016/j.jeconom.2007.08.002

Reference: ECONOM 2969

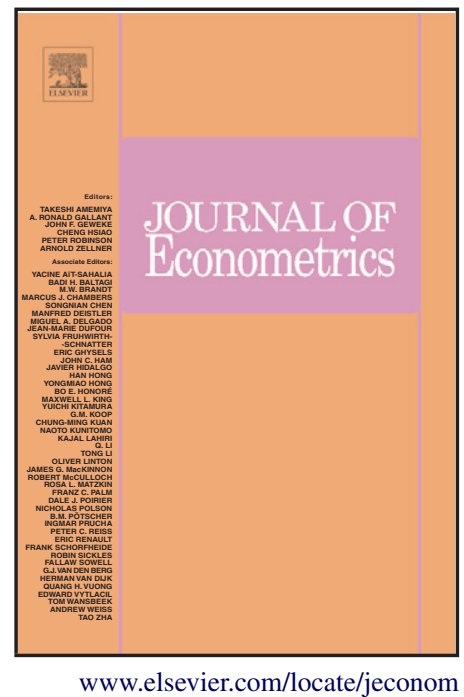

To appear in:

\section{Journal of Econometrics}

Cite this article as: Holger Dette and Mark Podolskij, Testing the parametric form of the volatility in continuous time diffusion models - a stochastic process approach, Journal of Econometrics (2007), doi:10.1016/j.jeconom.2007.08.002

This is a PDF file of an unedited manuscript that has been accepted for publication. As a service to our customers we are providing this early version of the manuscript. The manuscript will undergo copyediting, typesetting, and review of the resulting galley proof before it is published in its final citable form. Please note that during the production process errors may be discovered which could affect the content, and all legal disclaimers that apply to the journal pertain. 


\title{
Testing the parametric form of the volatility in continuous time diffusion models - a stochastic process approach
}

\author{
Holger Dette* \\ Mark Podolskij ${ }^{\dagger}$ \\ Ruhr-Universität Bochum \\ Ruhr-Universität Bochum \\ Fakultät für Mathematik \\ Fakultät für Mathematik
}

May 3, 2007

\begin{abstract}
We present new tests for the form of the volatility function which are based on stochastic processes of the integrated volatility. We prove weak convergence of these processes to centered processes whose conditional distributions are Gaussian. In the case of testing for a constant volatility the limiting process are standard Brownian bridges. As a consequence an asymptotic distribution free test and bootstrap tests (for testing of a general parametric form) can easily be implemented. It is demonstrated that the new tests are more than the currently available procedures. The new approach is also demonstrated by means of a simulation study.
\end{abstract}

Keywords and Phrases: Specification tests, integrated volatility, bootstrap, heteroscedasticity, stable convergence

*Holger Dette, Ruhr-Universität Bochum, Fakultät für Mathematik, 44780 Bochum, Germany, Tel: +49 2343228 284 ,FAX: +49 2343214 559, e-mail: holger.dette@rub.de

${ }^{\dagger}$ Mark Podolskij, Ruhr-Universität Bochum, Fakultät für Mathematik, 44780 Bochum, Germany, Tel: +49 234 3228330 , e-mail: podolski@cityweb.de 
Journal of Economic Literature Classification: C12

\section{Introduction}

Modeling the dynamics of interest rates, stock prices exchange rates is an important problem in mathematical finance and since the seminar papers of Black and Scholes (1973) and Merton (1973) many theoretical models have been developed for this purpose. Most of these models are continuous time stochastic processes, because information arrives at financial markets in continuous time (see Merton, 1990). A commonly used class of processes in mathematical finance for representing asset prices are Itô diffusions defined as a solution of the stochastic differential equation

$$
d X_{t}=b\left(t, X_{t}\right) d t+\sigma\left(t, X_{t}\right) d W_{t}
$$

where $\left(W_{t}\right)_{t}$ is a standard Brownian motion and $b$ and $\sigma$ denote the drift and volatility, respectively. Various models have been proposed in the literature for the different types of options (see e.g. Black and Scholes, 1973, Vasicek, 1977, Cox, Ingersoll and Ross, 1985, Karatzas, 1988, Constantinides, 1992 or Duffie and Harrison, 1993, among many others). For a reasonable pricing of derivative assets in the context of such models a correct specification of the volatility is required and good estimates of this quantity are needed. For example, the pricing of European call options crucially depends on the functional form of the volatility (see Black and Scholes, 1973) and the same is true for other types of options (see e.g. Duffie and Harrison, 1993, or Karatzas, 1988, among many others).

A (correct) specification of a parametric form for the volatility has the advantage that the problem of its estimation is reduced to the determination of a low dimensional parameter. On the other hand a misspecification of drift or variance in the diffusion model (1) may lead to an inadequate data analysis and to serious errors in the pricing of derivative assets. Therefore several authors propose to check the postulated model by means of a goodness-of-fit test (see Ait Sahalia, 1996, 
Corradi and White, 1999, Dette and von Lieres und Wilkau, 2003). Ait Sahalia (1996) assumes a time span approaching infinity as the sample size increases and considers the problem of testing a joint parametric specification of drift and variance, while in the other references a fixed time span is considered, where the discrete sampling interval approaches zero, and a parametric hypothesis regarding the volatility function is tested. This modeling might be more appropriate for high frequency data.

In the present paper we also consider the case of discretely observed data on a fixed time span, say $[0,1]$, from the model (1) with increasing sample size. As pointed out by Corradi and White (1999) this model is appropriate for analyzing the pricing of European, American or Asian options. These authors consider the sum of the squared differences between a nonparametric and a parametric estimate of the variance function at a fixed number of points in the interval $[0,1]$. Although this approach is attractive because of its simplicity, it has been argued by Dette and von Lieres und Wilkau (2003) that the results of the test may depend on the number and location of the points, where the parametric and nonparametric estimates are compared. Therefore these authors suggest a new test for the parametric form of the volatility in the diffusion model (1), which does not depend on the state $x$, i.e. $\sigma\left(t, X_{t}\right)=\sigma(t)$. The test is based on an $L^{2}$-distance between the volatility function in the model under the null hypothesis and alternative. This approach yields a consistent procedure against any (fixed) alternative, which can detect local alternatives converging to the null hypothesis at a rate $n^{-1 / 4}$. In the present paper an alternative test for the parametric form of the volatility function is proposed, which is based on a process of the integrated volatility. Our motivation for considering functionals of stochastic processes as test statistics stems from the fact that tests of this type are more sensitive with respect to Pitman alternatives. Moreover the new tests are also applicable for testing parametric hypotheses on the volatility, which depend on the state $x$.

In Section 2 we introduce some basic terminology and describe two kinds of parametric hypotheses for the volatility function. We also define two types of stochastic processes of the integrated 
volatility, which will be used for the construction of test statistics for these hypotheses. Section

3 contains our main results. We show convergence in probability of the stochastic processes to a random variable, which vanishes if and only if the null hypothesis is satisfied. Moreover, we also establish weak convergence of appropriately scaled processes of the integrated volatility to a centered process under the null hypothesis of a parametric form of the volatility. Consequently, the Kolmogorov-Smirnov and Cramér von Mises functional of these processes are natural test statistics. In general the limiting process is a complicated "function" of the data generating diffusion, but conditioned on the diffusion $\left(X_{t}\right)_{t \in[0,1]}$ it is a Gaussian process. In the problem of testing for homoscedasticity these tests are asymptotically distribution free and the limit distribution is given by a Brownian bridge. In Section 4 we study the finite sample properties of the proposed methodology and compare the new procedure with the currently available tests for the parametric form of the volatility function. For high frequency data the new tests yield a reliable approximation of the nominal level and substantial improvements with respect to power compared to the currently available procedures. Finally, all proofs and some auxiliary results are presented in an appendix.

\section{Specification of a parametric form of the volatility}

Let $\left(W_{t}\right)_{t \geq 0}$ denote a standard Brownian motion defined on an appropriate probability space $\left(\Omega, \mathcal{F},\left(\mathcal{F}_{t}\right)_{0 \leq t \leq 1}, P\right)$ with corresponding filtration $\mathcal{F}_{t}^{W}=\sigma\left(W_{s}, 0 \leq s \leq t\right)$ and assume that the drift and variance function in the stochastic differential equation (1) are locally Lipschitz continuous, i.e. for every integer $M>0$ there exists a constant $K_{M}$ such that

$$
|b(t, x)-b(t, y)|+|\sigma(t, x)-\sigma(t, y)| \leq K_{M}|x-y|
$$

for all $t \in[0,1], x, y \in[-M, M]$, and there exists a constant $K$ such that

$$
|b(t, x)|^{2}+|\sigma(t, x)|^{2} \leq K^{2}\left(1+|x|^{2}\right)
$$


for all $t \in[0,1], x \in \mathbb{R}$. Throughout this paper we assume additionally that the drift and variance function satisfy a further Lipschitz condition of order $\gamma>\frac{1}{2}$, i.e.

$$
|b(t, x)-b(s, x)|+|\sigma(t, x)-\sigma(s, x)| \leq L|t-s|^{\gamma}
$$

for some constant $L>0$. It is well known that for an $\mathcal{F}_{0}$-measurable square integrable random variable $\xi$, which is independent of the Brownian motion $\left(W_{t}\right)_{t \in[0,1]}$, the assumptions $(2)$ and (3) admit a unique strong solution $\left(X_{t}\right)_{t \in[0,1]}$ of the stochastic differential equation (1) with initial condition $X_{0}=\xi$ which is adapted to the filtration $\left(\mathcal{F}_{t}\right)_{0 \leq t \leq 1}$ (see e.g. Karatzas and Shreve, 1991, p. 289). The solution of the differential equation can be represented as

$$
X_{t}=\xi+\int_{0}^{t} b\left(s, X_{s}\right) d s+\int_{0}^{t} \sigma\left(s, X_{s}\right) d W_{s} \text { a.s. }
$$

where $X_{t}$ is $\mathcal{F}_{t}$-measurable for all $t \in[0,1]$ and the paths $t \rightarrow X_{t}$ are almost surely continuous. Throughout the paper we assume that

$$
E\left[|\xi|^{4}\right]<\infty
$$

and that the volatility $\sigma$ is twice continuously differentiable such that for some constant $F>0$

$$
\sup _{s, t \in[0,1]} E\left[\left(\frac{\partial}{\partial x} \sigma\left(s, X_{t}\right)\right)^{4}\right]<F, \sup _{s, t \in[0,1]} E\left[\left(\frac{\partial^{2}}{\partial x^{2}} \sigma\left(s, X_{t}\right)\right)^{4}\right]<F
$$

(here $\frac{\partial}{\partial x}$ denotes the partial derivative with respect to the second argument). In the literature various parametric functions have been proposed for different types of options (see e.g. Black and Scholes, 1973, Vasicek, 1977, Cox, Ingersoll and Ross, 1985, Karatzas, 1988, Constantinides, 1992 , or Duffie and Harrison, 1993, among many others). In principle the assumption on the volatility function in these models can be formulated in two ways that is

$$
\bar{H}_{0}: \sigma^{2}\left(t, X_{t}\right)=\sum_{j=1}^{d} \bar{\theta}_{j} \bar{\sigma}_{j}^{2}\left(t, X_{t}\right) \quad \forall t \in[0,1] \quad \text { (a.s.) },
$$

or

$$
H_{0}: \sigma\left(t, X_{t}\right)=\sum_{j=1}^{d} \theta_{j} \sigma_{j}\left(t, X_{t}\right) \quad \forall t \in[0,1] \quad \text { (a.s.) }
$$


where $\bar{\theta}=\left(\bar{\theta}_{1}, \ldots, \bar{\theta}_{d}\right), \theta=\left(\theta_{1}, \ldots, \theta_{d}\right) \in \Theta \subset \mathbb{R}^{d}$ are unknown finite dimensional parameters and $\bar{\sigma}_{1}^{2}, \ldots, \bar{\sigma}_{d}^{2}$, respectively $\sigma_{1}, \ldots, \sigma_{d}$ are given and known volatility functions satisfying

$$
\left|\bar{\sigma}_{j}(t, x)-\bar{\sigma}_{j}(s, x)\right|+\left|\sigma_{j}(t, x)-\sigma_{j}(s, x)\right| \leq L|t-s|^{\gamma}, \quad j=1, \ldots, d,
$$

for all $s, t \in[0,1], L>0, \gamma>\frac{1}{2}$. Note that the hypothesis (8) refers to the variance function $\sigma^{2}$ while the formulation (9) directly refers to the factor of the term $d W_{s}$ in the stochastic differential equation (1). There exist in fact many models for prices of financial assets traded in continuous time, where both hypotheses are equivalent (see e.g. Vasicek, 1977, Cox, Ingersoll and Ross, 1985, Brennan and Schwartz, 1979, Courtadon, 1982, Chan, Karolyi, Longstaff and Sanders, 1992), but in general these hypotheses are not equivalent. A typical example for such a case is given by

$$
\begin{array}{lll}
\bar{H}_{0}: & \sigma^{2}\left(t, X_{t}\right)=\bar{\vartheta}_{1}+\bar{\vartheta}_{2} X_{1}^{2}, \quad \text { (a.s.) } \\
H_{0}: & \sigma\left(t, X_{t}\right)=\vartheta_{1}+\vartheta_{2}\left|X_{t}\right|, \quad \text { (a.s.) }
\end{array}
$$

which is a slight generalization of the models considered in the cited references. We begin our discussion constructing a test statistic for the hypothesis $H_{0}$ in (9) and since the law of the process $X$ depends only on $\sigma^{2}$ (see Revuz and Yor, 1999, p. 293) we assume that the functions $\sigma_{1}, \ldots, \sigma_{d}$ are twice continuously differentiable, strictly positive, linearly independent on every compact set $[0,1] \times[a, b], a<b$ and satisfy the analogue of $(7)$, that is

$$
\sup _{s, t \in[0,1]} E\left[\left(\frac{\partial}{\partial x}\left\{\sigma_{i}\left(s, X_{t}\right) \sigma_{j}\left(s, X_{t}\right)\right\}\right)^{4}\right]<F, \sup _{s, t \in[0,1]} E\left[\left(\frac{\partial^{2}}{\partial x^{2}}\left\{\sigma_{i}\left(s, X_{t}\right) \sigma_{j}\left(s, X_{t}\right)\right\}\right)^{4}\right]<F
$$

for all $1 \leq i, j \leq d$. Further we assume that the functions $\sigma_{1}, \ldots, \sigma_{d}$ in the linear hypothesis (9) satisfy the same assumptions (2), (3) and (7) as the volatility function $\sigma$. For the discussion of hypothesis (8) we need to replace $\sigma$ by $\sigma^{2}$ in assumption (7), $\sigma_{i}$ by $\bar{\sigma}_{i}^{2}$ in (12) and 4 by 8 in assumption (6).

For testing the hypothesis (9) we consider the following stochastic process

$$
M_{t}:=\int_{0}^{t}\left\{\sigma\left(s, X_{s}\right)-\sum_{j=1}^{d} \theta_{j}^{m i n} \sigma_{j}\left(s, X_{s}\right)\right\} d s
$$


where the vector $\theta^{\text {min }}=\left(\theta_{1}^{\text {min }}, \ldots, \theta_{d}^{\text {min }}\right)^{T}:=\operatorname{argmin}_{\theta \in \mathbb{R}^{d}} \int_{0}^{1}\left\{\sigma\left(s, X_{s}\right)-\sum_{j=1}^{d} \theta_{j} \sigma_{j}\left(s, X_{s}\right)\right\}^{2} d s$. Note that the null hypothesis in (9) is satisfied if and only if $M_{t}=0 \forall t \in[0,1]$ (a.s.) and that we use an $L^{2}$-distance to determine the best approximation of $\sigma$ by linear combination of the functions $\sigma_{1}, \ldots, \sigma_{d}$. This choice is mainly motivated by the sake of transparency and other distances as the $L^{1}$-distance could be used as well with an additional amount of technical difficulties.

¿From standard Hilbert space theory (see Achieser, 1956) we obtain $\theta^{\text {min }}=D^{-1} C$, where the matrix $D=\left(D_{i j}\right)_{1 \leq i, j \leq d}$ and the vector $C=\left(C_{1}, \ldots, C_{d}\right)^{T}$ are defined by

$$
D_{i j}:=\left\langle\sigma_{i}, \sigma_{j}\right\rangle_{2}, C_{i}:=\left\langle\sigma, \sigma_{i}\right\rangle_{2}
$$

and $\langle\cdot, \cdot\rangle_{2}$ denotes the standard inner product for functions $f, g:[0,1] \times \mathbb{R} \rightarrow \mathbb{R}$, that is $\langle f, g\rangle_{2}=$ $\int_{0}^{1} f\left(t, X_{t}\right) g\left(t, X_{t}\right) d t$ (here and throughout this paper we assume that the integrals exist almost surely, whenever they appear in the text). The quantities in (14) can easily be estimated by

$$
\begin{aligned}
\hat{D}_{i j} & :=\frac{1}{n} \sum_{k=1}^{n} \sigma_{i}\left(\frac{k}{n}, X_{\frac{k}{n}}\right) \sigma_{j}\left(\frac{k}{n}, X_{\frac{k}{n}}\right) \stackrel{P}{\longrightarrow} D_{i j}, \\
\hat{C}_{i} & :=\mu_{1}^{-1} n^{-\frac{1}{2}} \sum_{k=1}^{n} \sigma_{i}\left(\frac{k-1}{n}, X_{\frac{k-1}{n}}\right)\left|X_{\frac{k}{n}}-X_{\frac{k-1}{n}}\right| \stackrel{P}{\longrightarrow} C_{i},
\end{aligned}
$$

where the symbol $\stackrel{P}{\longrightarrow}$ means convergence in probability and $\mu_{r}(r \geq 0)$ is defined as a the $r$ th absolute moment of a standard normal distribution (e.g. $\mu_{1}=\sqrt{2 / \pi}, \mu_{2}=1$ ). With the notation

$$
\hat{D}=\left(\hat{D}_{i j}\right)_{1 \leq i, j \leq d}, \hat{C}=\left(\hat{C}_{1}, \ldots, \hat{C}_{d}\right)^{T}
$$

we obtain $\hat{\theta}^{\text {min }}:=\hat{D}^{-1} \hat{C}$ as estimate for the random variable $\theta^{\text {min }}$. We finally introduce the stochastic process

$$
\hat{M}_{t}:=\hat{B}_{t}^{0}-\hat{B}_{t}^{T} \hat{D}^{-1} \hat{C}
$$

as estimate of the process $\left(M_{t}\right)_{t \in[0,1]}$ defined in (13), where the quantities $\hat{B}_{t}^{0}$ and $\hat{B}_{t}=\left(\hat{B}_{t}^{1}, \ldots, \hat{B}_{t}^{d}\right)^{T}$ are given by

$$
\hat{B}_{t}^{0}:=\mu_{1}^{-1} n^{-\frac{1}{2}} \sum_{k=1}^{\lfloor n t\rfloor}\left|X_{\frac{k}{n}}-X_{\frac{k-1}{n}}\right|, \quad \hat{B}_{t}^{i}:=\frac{1}{n} \sum_{k=1}^{\lfloor n t\rfloor} \sigma_{i}\left(\frac{k}{n}, X_{\frac{k}{n}}\right), \quad 1 \leq i \leq d,
$$


respectively. Because $M_{t}=0$ (a.s.) for all $t \in[0,1]$ if and only if the null hypothesis $H_{0}$ is satisfied, it is intuitively clear that the rejection of the null hypothesis for large values of $\sup _{t \in[0,1]}\left|\hat{M}_{t}\right|$, $\int_{0}^{1}\left|\hat{M}_{t}\right| d t$ or $\int_{0}^{1}\left|\hat{M}_{t}\right|^{2} d t$ yields a consistent test for the above problem. Note that the integrals and supremum have to be calculated with sufficient accuracy in order to avoid a discretization bias.

Before we make these arguments more rigorous we briefly present the corresponding testing procedure for the hypothesis (8). In this case the analogue of the stochastic process $M_{t}$ is defined by

$$
N_{t}:=\int_{0}^{t}\left\{\sigma^{2}\left(s, X_{s}\right)-\sum_{j=1}^{d} \bar{\theta}_{j}^{m i n} \bar{\sigma}_{j}^{2}\left(s, X_{s}\right)\right\} d s,
$$

where $\bar{\theta}^{\text {min }}=\left(\bar{\theta}_{1}^{\text {min }}, \ldots, \bar{\theta}_{d}^{\text {min }}\right)^{T}:=\operatorname{argmin}_{\bar{\theta} \in \mathbb{R}^{d}} \int_{0}^{1}\left\{\sigma^{2}\left(s, X_{s}\right)-\sum_{j=1}^{d} \bar{\theta}_{j} \bar{\sigma}_{j}^{2}\left(s, X_{s}\right)\right\}^{2} d s$. The nonobservable stochastic process can easily be estimated from the available data by

$$
\hat{N}_{t}:=\bar{B}_{t}^{0}-\bar{B}_{t}^{T} \bar{D}^{-1} \bar{C}
$$

where $\bar{D}_{i j}=1 / n \sum_{k=1}^{n} \bar{\sigma}_{i}^{2}\left(\frac{k}{n}, X_{\frac{k}{n}}\right) \bar{\sigma}_{j}^{2}\left(\frac{k}{n}, X_{\frac{k}{n}}\right)(i, j=1, \ldots, d), \bar{C}_{i}=\sum_{k=2}^{n} \bar{\sigma}_{i}^{2}\left(\frac{k-1}{n}, X_{\frac{k-1}{n}}\right)\left|X_{\frac{k}{n}}-X_{\frac{k-1}{n}}\right|^{2}$ $(i=1, \ldots, d)$ and the quantities $\bar{B}_{t}^{0}$ and $\bar{B}_{t}=\left(\bar{B}_{t}^{1}, \ldots \bar{B}_{t}^{d}\right)^{T}$ are given by

$$
\bar{B}_{t}^{0}:=\sum_{k=1}^{\lfloor n t\rfloor}\left|X_{\frac{k}{n}}-X_{\frac{k-1}{n}}\right|^{2}, \bar{B}_{t}^{i}:=\frac{1}{n} \sum_{k=1}^{\lfloor n t\rfloor} \bar{\sigma}_{i}^{2}\left(\frac{k}{n}, X_{\frac{k}{n}}\right), \quad 1 \leq i \leq d .
$$

In the following section we investigate the stochastic properties of the processes $\left(\sqrt{n}\left(\hat{M}_{t}-M_{t}\right)\right)_{t \in[0,1]}$ and $\left(\sqrt{n}\left(\hat{N}_{t}-N_{t}\right)\right)_{t \in[0,1]}$. In particular, we will prove weak convergence of these processes to centered processes, which are conditioned on the process $\left(X_{t}\right)_{t \in[0,1]}$ Gaussian processes. This is the basic result for the application of these processes in the problem of testing for the parametric form of the volatility in a continuous time diffusion model. The reason for considering both processes is twofold. On the one hand the weak convergence of the process $\sqrt{n}\left(\hat{M}_{t}-M_{t}\right)$ can be established under weaker assumptions on the model (1). On the other hand the statistic $\sqrt{n}\left(\hat{N}_{t}-N_{t}\right)$ can easily be extended to vector-valued diffusions (see Remark 3.7). 


\section{Main results}

For the sake of brevity we mainly restrict ourselves to a detailed derivation of the stochastic properties of the process $\hat{M}_{t}$. The corresponding results for the process $\hat{N}_{t}$ can be obtained by similar arguments and the main statements are given at the end of this section for the sake of completeness. We begin our discussion with two auxiliary results regarding the estimators $\hat{D}$ and $\hat{B}_{t}^{i}$ defined in (17) and (19), which are also of own interest. Our first results clarify the order of difference between the empirical quantities $\hat{C}_{i}, \hat{B}_{t}^{i}, \hat{D}_{i j}$ and their theoretical counterparts $C_{i}, B_{t}^{i}, D_{i j}$, respectively.

Lemma 3.1. If the assumptions stated in Section 2 are satisfied we have $\hat{D}-D=o_{p}\left(n^{-\frac{1}{2}}\right)$ and

$$
\hat{B}_{t}^{i}-\int_{0}^{t} \sigma_{i}\left(s, X_{s}\right) d s=o_{p}\left(n^{-\frac{1}{2}}\right) \quad 1 \leq i \leq d
$$

Throughout this paper the symbol $X_{n} \stackrel{\mathcal{D}_{s t}}{\longrightarrow} X$ means that the sequence of random variables converges stably in law. Recall that a sequence of $d$-dimensional random variables $\left(X_{n}\right)_{n \in \mathbb{N}}$ converges stably in law with limit $X$, defined on an appropriate extension $\left(\Omega^{\prime}, \mathcal{F}^{\prime}, P^{\prime}\right)$ of a probability space $(\Omega, \mathcal{F}, P)$, if and only if for any $\mathcal{F}$-measurable and bounded random variable $Y$ and any bounded and continuous function $g$ the convergence $\lim _{n \rightarrow \infty} E\left[Y g\left(X_{n}\right)\right]=E[Y g(X)]$ holds. This is obviously a slightly stronger mode of convergence than convergence in law (see Renyi, 1963, Aldous and Eagleson, 1978, for more details on stable convergence). The following Lemma shows that the random variables $\hat{B}_{t}^{0}$ and $\hat{C}_{i}$ defined in (19) and (17) converge stably in law if they are appropriately normalized.

Lemma 3.2. If the assumptions stated in Section 2 are satisfied we have for any $t_{1}, \ldots, t_{k} \in[0,1]$

$$
\sqrt{n}\left(\begin{array}{c}
\left(\hat{B}_{t_{\ell}}^{0}-<\sigma, 1>_{2}^{t_{\ell}}\right)_{\ell=1}^{k} \\
\left(\hat{C}_{\ell}-C_{\ell}\right)_{\ell=1}^{d}
\end{array}\right) \stackrel{\mathcal{D}_{s t}}{\longrightarrow} \mu_{1}^{-1} \sqrt{\mu_{2}-\mu_{1}^{2}} \int_{0}^{1} \Sigma_{t_{1}, \ldots t_{k}}^{\frac{1}{2}}\left(s, X_{s}\right) d W_{s}^{\prime},
$$

where $W^{\prime}$ denotes a $(d+k)$-dimensional Brownian motion, which is independent of the $\sigma$-field $\mathcal{F}$, and the matrix $\Sigma_{t_{1}, \ldots t_{k}}\left(s, X_{s}\right)$ denotes a block matrix defined by

$$
\Sigma_{t_{1}, \ldots t_{k}}\left(s, X_{s}\right)=\left(\begin{array}{cc}
E & F \\
F^{T} & G
\end{array}\right)
$$


with

$$
\begin{aligned}
E & =\left(\sigma^{2}\left(s, X_{s}\right) 1_{\left[0, t_{i} \wedge t_{j}\right)}(s)\right)_{i=1, \ldots, k}^{j=1, \ldots, k}, F=\left(\sigma_{j}\left(s, X_{s}\right) \sigma^{2}\left(s, X_{s}\right) 1_{\left[0, t_{i}\right)}(s)\right)_{i=1, \ldots, k}^{j=1, \ldots, d} \\
G & =\left(\sigma_{i}\left(s, X_{s}\right) \sigma_{j}\left(s, X_{s}\right) \sigma^{2}\left(s, X_{s}\right)\right)_{i=1, \ldots, d}^{j=1, \ldots, d}
\end{aligned}
$$

and $<\sigma, 1>_{2}^{t}=\int_{0}^{t} \sigma\left(s, X_{s}\right) d s$.

Note that the matrix $\Sigma_{t_{1}, \ldots t_{k}}\left(s, X_{s}\right)$ defined in (24) can be represented as

$$
\Sigma_{t_{1}, \ldots, t_{k}}^{1 / 2}\left(s, X_{s}\right)=\frac{g_{t_{1}, \ldots, t_{k}}\left(s, X_{s}\right) g_{t_{1}, \ldots, t_{k}}\left(s, X_{s}\right)^{T}}{\sqrt{g_{t_{1}, \ldots, t_{k}}\left(s, X_{s}\right)^{T} g_{t_{1}, \ldots, t_{k}}\left(s, X_{s}\right)}}
$$

where the vector $g_{t_{1}, \ldots, t_{k}}\left(s, X_{s}\right)$ is defined by

$$
g_{t_{1}, \ldots, t_{k}}\left(s, X_{s}\right)=\left(\sigma\left(s, X_{s}\right)\left\{I_{\left[0, t_{1}\right)}(s), \ldots, I_{\left[0, t_{k}\right)}(s)\right\}, \sigma\left(s, X_{s}\right)\left\{\sigma_{1}\left(s, X_{s}\right), \ldots, \sigma_{d}\left(s, X_{s}\right)\right\}\right)^{T}
$$

Now we state one of our main results.

Theorem 3.3. If the assumptions given in Section 2 are satisfied, then the process $\left\{A_{n}(t)\right\}_{t \in[0,1]}=$ $\left\{\sqrt{n}\left(\hat{M}_{t}-M_{t}\right)\right\}_{t \in[0,1]}$ converges weakly on $D[0,1]$ to a process $\{A(t)\}_{t \in[0,1]}$, which is Gaussian conditioned on the $\sigma$-field $\mathcal{F}$. Moreover, the finite dimensional conditional distributions of the limiting process $\left(A\left(t_{1}\right), \ldots A\left(t_{k}\right)\right)^{T}$ are uniquely determined by the conditional covariance matrix

$$
\mu_{1}^{-2}\left(\mu_{2}-\mu_{1}^{2}\right) V \int_{0}^{1} \Sigma_{t_{1}, \ldots t_{k}}\left(s, X_{s}\right) d s V^{T}
$$

where the $k \times(d+k)$-dimensional matrix $V$ is defined by $V=\left(I_{k} \mid \tilde{V}\right), \tilde{V}=-\left(B_{t_{\ell}}^{T} D^{-1}\right)_{\ell=1, \ldots d}$ and $I_{k} \in \mathbb{R}^{k \times k}$ denotes the identity matrix.

Note that the identity $M_{t} \equiv 0$ holds (a.s.) for all $t \in[0,1]$ if and only if the null hypothesis in (9) is satisfied, and consequently the null hypothesis is rejected for large values of a functional of the process $\left(\sqrt{n} \hat{M}_{t}\right)_{t \in[0,1]}$. For example, in the case of the Kolmogorov-Smirnov statistic $K_{n}=$ $\sqrt{n} \sup _{t \in[0,1]}\left|\hat{M}_{t}\right|$, it follows from Theorem 3.3 that (under $\left.H_{0}\right) K_{n} \stackrel{\mathcal{D}}{\longrightarrow} \sup _{t \in[0,1]}|A(t)|$, where the symbol $\stackrel{\mathcal{D}}{\longrightarrow}$ denotes the weak convergence and the process $(A(t))_{t \in[0,1]}$ is defined in Theorem 3.3. In 
general, even under the null hypothesis $H_{0}$, the distribution of this process is rarely available and depends on the full process $\left(X_{t}\right)_{t \in[0,1]}$. However, conditioned on the process $\left(X_{t}\right)_{t \in[0,1]}$ the process $(A(t))_{t \in[0,1]}$ is Gaussian. Moreover, in the important case of testing for a constant volatility, i.e. $d=1, \sigma_{1}\left(t, X_{t}\right)=1$, the limit distribution of the process $\left(A_{n}(t)\right)_{t \in[0,1]}$ is surprisingly simple.

Corollary 3.4. Assume that the assumptions stated in Section 2 are satisfied and that the hypothesis $H_{0}: \sigma\left(t, X_{t}\right)=\sigma$ has to be tested (that is $d=1, \sigma_{1}\left(t, X_{t}\right)=1$ in (9)). Under the null hypothesis the process $\left(A_{n}(t)\right)_{t \in[0,1]}$ converges weakly on $D[0,1]$ to the process $\left(\mu_{1}^{-1} \sqrt{\mu_{2}-\mu_{1}^{2}} \sigma B_{t}^{\prime}\right)_{t \in[0,1]}$, where $B_{t}^{\prime}$ denotes a Brownian bridge.

We now briefly consider the corresponding results for testing the hypothesis (8) based on the stochastic process $\hat{N}_{t}$ defined in (21). The following result is proved by similar arguments as presented for the proof of Theorem 3.3 in the Appendix.

Theorem 3.5. If the assumptions given in Section 2 are satisfied, then the process $\left\{\bar{A}_{n}(t)\right\}_{t \in[0,1]}=$ $\left\{\sqrt{n}\left(\hat{N}_{t}-N_{t}\right)\right\}_{t \in[0,1]}$ converges weakly on $D[0,1]$ to a process $(\bar{A}(t))_{t \in[0,1]}$, which is Gaussian conditioned on the $\sigma$-field $\mathcal{F}$. Moreover, the finite dimensional conditional distributions of the limiting process $\left(\bar{A}\left(t_{1}\right), \ldots \bar{A}\left(t_{k}\right)\right)^{T}$ are uniquely determined by the conditional covariance matrix

$$
2 \bar{V} \int_{0}^{1} \bar{\Sigma}_{t_{1}, \ldots t_{k}}\left(s, X_{s}\right) d s \bar{V}^{T},
$$

where the $k \times(d+k)$-dimensional matrix $\bar{V}$ is defined by $\bar{V}=\left(I_{k} \mid \tilde{U}\right), \tilde{U}=-\left(\bar{B}_{t_{\ell}}^{T} \bar{D}^{-1}\right)_{\ell=1, \ldots k}$, and the matrix $\bar{\Sigma}_{t_{1}, \ldots t_{k}}$ is given by

$$
\bar{\Sigma}_{t_{1}, \ldots t_{k}}\left(s, X_{s}\right)=\left(\begin{array}{cc}
\bar{E} & \bar{F} \\
\bar{F}^{T} & \bar{G}
\end{array}\right)
$$

with

$$
\begin{array}{ll}
\bar{E}=\left(\sigma^{4}\left(s, X_{s}\right) 1_{\left[0, t_{i} \wedge t_{j}\right)}(s)\right)_{i=1, \ldots, k}^{j=1, \ldots, k}, & \bar{F}=\left(\bar{\sigma}_{j}^{2}\left(s, X_{s}\right) \sigma^{4}\left(s, X_{s}\right) 1_{\left[0, t_{i}\right)}(s)\right)_{i=1, \ldots, k}^{j=1, \ldots, d} \\
\bar{G}=\left(\bar{\sigma}_{i}^{2}\left(s, X_{s}\right) \bar{\sigma}_{j}^{2}\left(s, X_{s}\right) \sigma^{4}\left(s, X_{s}\right)\right)_{i=1, \ldots, d}^{j=1, \ldots, d}, & \bar{B}_{t}=\left(\int_{0}^{t} \bar{\sigma}_{1}^{2}\left(s, X_{s}\right) d s, \ldots, \int_{0}^{t} \bar{\sigma}_{d}^{2}\left(s, X_{s}\right) d s\right)^{T} .
\end{array}
$$


We proceed this section with an investigation of the stochastic properties of the tests with respect to local alternatives. For the sake of brevity we restrict ourselves to the problem of testing for homoscedasticity, that is $H_{0}: \sigma\left(t, X_{t}\right)=\sigma$ a.s. for some $\sigma>0$. The problem of testing more general hypotheses can be treated exactly in the same way. The consideration of the null hypothesis of homoscedasticity additionally allows a comparison of the two approaches based on Theorem 3.3 and 3.5 , because in the special case $d=1$ the hypotheses (8) and (9) are in fact equivalent. Note that the process corresponding to the hypothesis $H_{0}: \sigma\left(t, X_{t}\right)=\sigma$ is given by

$$
\hat{M}_{t}=\mu_{1}^{-1} n^{-\frac{1}{2}}\left(\sum_{k=2}^{\lfloor n t\rfloor}\left|X_{\frac{k}{n}}-X_{\frac{k-1}{n}}\right|-\frac{\lfloor n t\rfloor}{n} \sum_{k=2}^{n}\left|X_{\frac{k}{n}}-X_{\frac{k-1}{n}}\right|\right)
$$

Similarly, if the process defined by $(21)$ is used we have (in the case $d=1, \sigma_{1}\left(s, X_{s}\right)=1$ )

$$
\hat{N}_{t}=\sum_{k=2}^{\lfloor n t\rfloor}\left(X_{\frac{k}{n}}-X_{\frac{k-1}{n}}\right)^{2}-\frac{\lfloor n t\rfloor}{n} \sum_{k=2}^{n}\left(X_{\frac{k}{n}}-X_{\frac{k-1}{n}}\right)^{2} .
$$

We finally also introduce the statistic proposed by Dette and von Lieres und Wilkau (2003) for the hypothesis of homoscedasticity, that is

$$
\hat{G}=\sqrt{n}\left\{\frac{n}{3} \sum_{k=2}^{n}\left(X_{\frac{k}{n}}-X_{\frac{k-1}{n}}\right)^{4}-\left(\sum_{k=2}^{n}\left(X_{\frac{k}{n}}-X_{\frac{k-1}{n}}\right)^{2}\right)^{2}\right\}
$$

The following results specify the asymptotic behaviour of the processes $\hat{M}_{t}, \hat{N}_{t}$ and the statistic $\hat{G}$ under local alternatives.

Theorem 3.6. Consider local alternatives of the form $H_{1}^{(n)}: \sigma\left(t, X_{t}\right)=\sigma+\gamma_{n} h\left(t, X_{t}\right)$, where $h$ is a positive function and $\gamma_{n}$ is a positive sequence converging to 0 .

(a) If the assumptions of Theorem 3.3 are satisfied, $\gamma_{n}=n^{-1 / 2}$, then the processes $\left(\sqrt{n} \hat{M}_{t}\right)_{t \in[0,1]}$ defined in (29) converges weakly on $D[0,1]$ to the process $\left(\mu_{1}^{-1} \sqrt{\mu_{2}-\mu_{1}^{2}} \sigma B_{t}^{\prime}+R_{t}\right)_{t \in[0,1]}$, where $B_{t}^{\prime}$ denotes a Brownian bridge, the process $R_{t}$ is given by

$$
R_{t}=\left(\int_{0}^{t} h\left(s, X_{s}\right) d s-t \int_{0}^{1} h\left(s, X_{s}\right) d s\right)
$$

and the processes $\left(B_{t}^{\prime}\right)_{t \in[0,1]}$ and $\left(R_{t}\right)_{t \in[0,1]}$ are stochastically independent. 
(b) If the assumptions of Theorem 3.5 are satisfied, $\gamma_{n}=n^{-1 / 2}$, then the process $\left(\sqrt{n} \hat{N}_{t}\right)_{t \in[0,1]}$ defined by (30) converges weakly on $D[0,1]$ to the process $\left(\sqrt{2} \sigma^{2} B_{t}^{\prime}+2 \sigma R_{t}\right)_{t \in[0,1]}$, where $B_{t}^{\prime}$ denotes a Brownian bridge, the process $R_{t}$ is given in (32) and the processes $\left(B_{t}^{\prime}\right)_{t \in[0,1]}$ and $\left(R_{t}\right)_{t \in[0,1]}$ are stochastically independent.

(c) If the assumptions of Theorem 3.5 are satisfied, $\gamma_{n}=n^{-1 / 4}$, then it follows for the random variable $\hat{G}$ defined in (31)

$$
\sqrt{n} \hat{G} \stackrel{\mathcal{D}}{\longrightarrow} Z+4 \sigma^{2}\left(\int_{0}^{1} h^{2}\left(s, X_{s}\right) d s-\left(\int_{0}^{1} h\left(s, X_{s}\right) d s\right)^{2}\right),
$$

where the random variables $Z \sim \mathcal{N}\left(0, \frac{8}{3} \sigma^{8}\right)$ and $\left(\int_{0}^{1} h^{2}\left(s, X_{s}\right) d s-\left(\int_{0}^{1} h\left(s, X_{s}\right) d s\right)^{2}\right)$ are stochastically independent

Note that it follows from Theorem 3.6 that goodness-of-fit tests based on the processes (29) and (30) are more powerful with respect to the Pitman alternatives considered in Theorem 3.6 than the test which rejects the null hypothesis of homoscedasticity for large values of the statistic $\hat{G}$. Moreover, Theorem 3.6 also shows that there will be no substantial differences between the tests based on the stochastic processes $\hat{M}_{t}$ and $\hat{N}_{t}$ with respect to power for the local alternatives considered in Theorem 3.6 (besides that the asymptotic theory for the latter requires slightly stronger assumptions). We finally note again that a similar statement can be shown for the general hypotheses (8) and (9).

Remark 3.7. It is worthwhile to mention that the process $\left(N_{t}\right)_{t \in[0,1]}$ can easily be generalized to $p$ dimensional diffusions. For this assume that the drift function $b$ in (1) is a $p$-dimensional vector, the volatility is a $p \times q$ matrix, and the underlying Brownian motion is $q$-dimensional. For functions $f, g$ : $[0,1] \times \mathbb{R}^{p} \rightarrow \mathbb{R}^{p \times p}$ we define the (random) inner product $\langle f, g\rangle_{2}=\int_{0}^{1} \operatorname{trace}\left(f\left(s, X_{s}\right) g\left(s, X_{s}\right)^{T}\right) d s$ and denote by $\bar{\theta}^{\text {min }}:=\operatorname{argmin}_{\bar{\theta} \in \mathbb{R}^{d}}\left\langle\sigma \sigma^{T}-\sum_{j=1}^{d} \bar{\theta}_{j} \bar{\sigma}_{j} \bar{\sigma}_{j}^{T}, \sigma \sigma^{T}-\sum_{j=1}^{d} \bar{\theta}_{j} \bar{\sigma}_{j} \bar{\sigma}_{j}^{T}\right\rangle_{2}=\bar{D}^{-1} \bar{C}$, where the elements of the matrix $\bar{D}=\left(\bar{D}_{i j}\right)_{1 \leq i, j \leq d}$ and the vector $\bar{C}=\left(\bar{C}_{1}, \ldots, \bar{C}_{d}\right)^{T}$ are defined as $\bar{D}_{i j}:=\left\langle\bar{\sigma}_{i} \bar{\sigma}_{i}^{T}, \bar{\sigma}_{j} \bar{\sigma}_{j}^{T}\right\rangle_{2}$ , $\bar{C}_{i}:=\left\langle\sigma \sigma^{T}, \bar{\sigma}_{i} \bar{\sigma}_{i}^{T}\right\rangle_{2}$. Finally we define the $p \times p$ process

$$
N_{t}:=\int_{0}^{t}\left\{\sigma\left(s, X_{s}\right) \sigma\left(s, X_{s}\right)^{T}-\sum_{j=1}^{d} \bar{\theta}_{j}^{m i n} \bar{\sigma}_{j}\left(s, X_{s}\right) \bar{\sigma}_{j}\left(s, X_{s}\right)^{T}\right\} d s,
$$


then it is easy to see that the null hypothesis $\sigma \sigma^{T}=\sum_{j=1}^{d} \bar{\theta}_{j} \bar{\sigma}_{j} \bar{\sigma}_{j}^{T}$ is valid if and only if $M_{t} \equiv 0$ $\forall t \in[0,1]$ (a.s.). This process is now estimated in an obvious way. For example, the first term in (33) can be approximated by the data by

$$
\sum_{i=1}^{[n t]}\left(X_{\frac{i}{n}}-X_{\frac{i-1}{n}}\right)\left(X_{\frac{i}{n}}-X_{\frac{i-1}{n}}\right)^{T} \stackrel{P}{\longrightarrow} \int_{0}^{t} \sigma\left(s, X_{s}\right) \sigma\left(s, X_{s}\right)^{T} d s,
$$

and the other terms are treated similarly. Consequently under appropriate assumptions on the drift $b$ the volatility $\sigma$ and the functions $\bar{\sigma}_{1}, \ldots \bar{\sigma}_{d}$ analogues of Theorem 3.5 and 3.6 are available for the vector-valued diffusions.

We conclude this section with a corresponding result for a standardized version of the process $A_{n}(t)$ (a similar result holds for $\bar{A}_{n}(t)$ and is omitted for the sake of brevity). Observe that the asymptotic conditional variance of $A_{n}(t)$ is given by

$$
s_{t}^{2}=\mu_{1}^{-2}\left(\mu_{2}-\mu_{1}^{2}\right)\left(1,-B_{t}^{T} D^{-1}\right) \int_{0}^{1} \Sigma_{t}\left(s, X_{s}\right) d s\left(1,-B_{t}^{T} D^{-1}\right)^{T}
$$

For the corresponding estimate, say $\hat{s}_{t}^{2}$, the random variables $B_{t}$ und $D$ are replaced by their empirical counterparts, and the random elements in the matrix $\Sigma_{t}\left(s, X_{s}\right)$ defined in $(24)$ are replaced by the statistics

$$
\begin{aligned}
& \sum_{k=1}^{[n t]}\left(X_{\frac{k}{n}}-X_{\frac{k-1}{n}}\right)^{2} \stackrel{P}{\longrightarrow} \int_{0}^{t} \sigma^{2}\left(s, X_{s}\right) d s \\
& \sum_{k=1}^{[n t]} \sigma_{i}^{2}\left(\frac{k-1}{n}, X_{\frac{k-1}{n}}\right)\left(X_{\frac{k}{n}}-X_{\frac{k-1}{n}}\right)^{2} \stackrel{P}{\longrightarrow} \int_{0}^{t} \sigma_{i}^{2}\left(s, X_{s}\right) \sigma^{2}\left(s, X_{s}\right) d s \\
& \sum_{k=1}^{n} \sigma_{j}^{2}\left(\frac{k-1}{n}, X_{\frac{k-1}{n}}\right) \sigma_{i}^{2}\left(\frac{k-1}{n}, X_{\frac{k-1}{n}}\right)\left(X_{\frac{k}{n}}-X_{\frac{k-1}{n}}\right)^{2} \stackrel{P}{\longrightarrow} \int_{0}^{1} \sigma_{j}^{2}\left(s, X_{s}\right) \sigma_{i}^{2}\left(s, X_{s}\right) \sigma^{2}\left(s, X_{s}\right) d s
\end{aligned}
$$

For the final result of this section we require the following non-singularity condition

$$
\text { The matrix } \int_{0}^{1} \Sigma_{t}\left(s, X_{s}\right) d s \text { is positive definite for all } t \in[\underline{x}, \bar{x}], 0<\underline{x}<\bar{x} \leq 1 \text {. }
$$

Note that $\int_{0}^{1} \Sigma_{0}\left(s, X_{s}\right) d s$ is singular. Furthermore, when $d=1, \sigma_{1}\left(t, X_{t}\right)=1$ in (9) the matrix $\int_{0}^{1} \Sigma_{1}\left(s, X_{s}\right) d s$ is also singular $($ so $\bar{x}<1)$. 
Theorem 3.8. If the assumptions given in Section 2 and condition (35) are satisfied, then the process $\left(A_{n}(t) / \hat{s}_{t}\right)_{t \in[\underline{x}, \bar{x}]}$ converges weakly on $D[0,1]$ to a process $\left(A(t) / s_{t}\right)_{t \in[\underline{x}, \bar{x}]}$, where $(A(t))_{t \in[0,1]}$ is defined in Theorem 3.3.

\section{$4 \quad$ Finite sample properties}

In this section we investigate the finite sample properties of Kolmogorov-Smirnov tests based on the processes $\left(\hat{M}_{t}\right)_{t \in[0,1]}$ and $\left(\hat{N}_{t}\right)_{t \in[0,1]}$. We also compare these tests with the test, which was recently proposed by Dette, Podolskij and Vetter (2005) for the hypotheses of the form (8). We begin with a study of the quality of approximation by a Brownian bridge in the case of testing for homoscedasticity. In the second part of this section we briefly investigate the performance of a parametric bootstrap procedure for the problem of testing more general hypotheses and present an example analyzing exchange rate data. Here and throughout this section all reported results are based on 1000 simulation runs. Note that this number of runs still produces some variability in the simulated results.

4.1 Testing for homoscedasticity. Recall from Corollary 3.4 that under the null hypothesis

$$
M^{(n)}:=\sqrt{n} \sup _{t \in[0,1]}\left|\frac{\hat{M}_{t}}{\hat{\beta}}\right| \stackrel{\mathcal{D}}{\longrightarrow} \sup _{t \in[0,1]}\left|B_{t}^{\prime}\right|
$$

where $\left(B_{t}^{\prime}\right)_{t \in[0,1]}$ denotes a Brownian bridge and $\hat{\beta}=\mu_{1}^{-2} \sqrt{\mu_{2}-\mu_{1}^{2}} n^{-1 / 2} \sum_{i=2}^{n}\left|X_{\frac{i}{n}}-X_{\frac{i-1}{n}}\right|$. Similarly, it follows from Theorem 3.5 that

$$
N^{(n)}:=\sqrt{n} \sup _{t \in[0,1)}\left|\frac{\hat{N}_{t}}{\hat{\gamma}}\right| \stackrel{\mathcal{D}}{\longrightarrow} \sup _{t \in[0,1]}\left|B_{t}^{\prime}\right|
$$

where the process $\left(\hat{N}_{t}\right)_{t \in[0,1]}$ is defined in (30) and $\hat{\gamma}=\sqrt{2} \sum_{i=1}^{n}\left(X_{\frac{i}{n}}-X_{\frac{i-1}{n}}\right)^{2}$. The null hypothesis of a constant volatility in the stochastic differential equation is now rejected if $M^{(n)}$ or $N^{(n)}$ exceed the corresponding quantile of the distribution of the maximum of a Brownian bridge on the interval $[0,1]$. In Table 1 we show the approximation of the nominal level of these tests for sample sizes 
$n=100,200,500$. The data was generated according to the diffusion model (1) with $\sigma=1$ and various drift functions $b(t, x)$.

\section{Insert Table 1}

We observe a reasonable approximation of the nominal level in most cases. The statistic $M^{(n)}$ usually yields a more precise approximation of the nominal level than the statistic $N^{(n)}$, which turns out to be slightly conservative for small sample sizes. We now investigate the power of both tests in the problem of testing for homoscedasticity. For the sake of comparison we consider the same scenario as in Dette, von Lieres und Wilkau (2003) who proposed the test based on the statistic $\hat{G}$ in (31) for the problem of checking homoscedasticity. Following these authors we chose the volatitlity functions $\sigma(t, x)=1+x, 1+\sin (5 x), 1+x e^{t}, 1+x \sin (5 t), 1+x t$. In Table 2 we present the corresponding rejection probabilities for the sample sizes $n=100,200$ and 500 . The results are directly comparable with the results in Table 3 of Dette and von Lieres und Wilkau (2003) for the corresponding test based on the statistic (31). From Theorem 3.6 we expect some improvement in local power with respect to Pitman alternatives by the new procedure and these theoretical advantages are impressively reflected in our simulation study. We observe a substantial increase in power for the new tests. In most cases the improvement is at least approximately $15 \%$ and there are many cases, for which the power of the new test with 200 observations already exceeds the power of the test of Dette, von Lieres und Wilkau (2003) for 500 observations.

\section{Insert Table 2}

4.2 Testing for the parametric form of the volatility. As pointed out previously, for a general null hypothesis the asymptotic distribution of the processes depends on the underlying diffusion $\left(X_{t}\right)_{t \in[0,1]}$ and cannot be used for the calculation of critical values (except in the problem of testing for homoscedasticity). However, conditional on $\left(X_{t}\right)_{t \in[0,1]}$ the limiting processes in Theorem 3.3, 3.5 and 3.8 are Gaussian and this suggests that the parametric bootstrap can be used to obtain critical 
values. In this paragraph we will investigate the finite sample performance of this approach. We explain this procedure for the process $\left(\hat{M}_{t}\right)_{t \in[0,1]}$, the corresponding bootstrap test for the process $\left(\hat{N}_{t}\right)_{t \in[0,1]}$ is obtained similarly. We consider the standardized Kolmogorov-Smirnov statistic

$$
Z_{n}=\sup \left\{\left|\sqrt{n} \hat{M}_{t} / \hat{s}_{t}\right| \mid t \in[0,1]\right\}
$$

Then the least squares estimator $\hat{\theta}^{\mathrm{min}}=\left(\hat{\theta}_{1}^{\mathrm{min}}, \ldots, \hat{\theta}_{d}^{\mathrm{min}}\right)^{T}$ is determined and the data $X_{i / n}^{*(j)}(i=$ $1, \ldots, n ; j=1, \ldots B)$ from the stochastic differential equation (1) with $b(t, x) \equiv 0$ and $\sigma(t, x)=$ $\sum_{j=1}^{d} \hat{\theta}_{j}^{\min } \sigma_{j}(t, x)$ are generated (note that this choice corresponds to the null hypothesis (9)). These data are used to calculate the bootstrap analogues $Z_{n}^{*(1)}, \ldots, Z_{n}^{*(B)}$ of the statistic $Z_{n}$ defined in (38). Finally the value of the statistic $Z_{n}$ is compared with the corresponding quantiles of the bootstrap distribution.

We have investigated the performance of this resampling procedure for the problem of testing various linear hypotheses, where the volatility function depends on the variable $x$. The sample sizes are again $n=100,200,500$ and $B=500$ bootstrap replications are used for the calculation of the critical values. In particular we compare the two procedures based on $\left(\hat{M}_{t}\right)_{t \in[0,1]}$ and $\left(\hat{N}_{t}\right)_{t \in[0,1]}$ for testing the hypothesis

$$
\bar{H}_{0}: \sigma^{2}(t, x)=\bar{\theta} x^{2}, \quad H_{0}: \sigma(t, x)=\theta x
$$

In Table 3 we display the simulated level of the parametric bootstrap tests for various drift functions. We observe a better approximation of the nominal level by the test based on the process $\left(N_{t}\right)_{t \in[0,1]}$, in particular for small sample sizes. The Kolmogorov-Smirnov test based on the process $\left(\hat{M}_{t}\right)_{t \in[0,1]}$ yields a reliable approximation of the nominal level for sample sizes larger than 200, while the statistic based on the process $\hat{N}_{t}$ can already be recommended for $n=100$. The results for sample size $n=200,500$ demonstrate that for high frequency data as considered in this paper both tests yield a reliable approximation of the nominal level. In Table 4 we show the simulated rejection probabilities for the case $b(t, x)=2-x$ and the alternatives $\sigma^{2}(t, x)=1+x^{2}, 1,5|x|^{3 / 2}, 5|x|$, $(1+x)^{2}$ 


\section{Insert Table 3 and 4}

Note that the Kolmogorov-Smirnov test based on the process $\left(\hat{M}_{t}\right)_{t \in[0,1]}$ is substantially more powerful than the test based on the process $\left(\hat{N}_{t}\right)_{t \in[0,1]}$ which uses the squared differences. This superiority is partially bought by a worse approximation of the nominal level for smaller sample sizes (see the results for $n=100$ and $n=200$ in Table 3). However, in the case $b(t, x)=2-x$ and $n=200,500$ both tests keep approximately their level, but the test based on $\left(\hat{M}_{t}\right)_{t \in[0,1]}$ is still substantially more powerful. Thus for high frequency data we recommend the application of the Kolmogorov-Smirnov test based on the process $\left(\hat{M}_{t}\right)_{t \in[0,1]}$.

It is also of interest to compare the power of the new tests with a bootstrap test, which was recently proposed by Dette, Podolskij and Vetter (2005) and is based on an estimate of the $L^{2}$ distance $M^{2}=\min _{\theta_{1}, \ldots, \theta_{d}} \int_{0}^{1}\left\{\sigma^{2}\left(t, X_{t}\right)-\sum_{j=1}^{d} \theta_{j} \sigma_{j}^{2}\left(t, X_{t}\right)\right\}^{2} d t$. Because this test yield a rather accurate approximation of the nominal level (see Table 1 in this reference) we mainly consider the Kolmogorov-Smirnov test based on the process $\left(\hat{N}_{t}\right)_{t \in[0,1]}$ in our comparison. The results in the right part of Table 4 are directly comparable with the results displayed in Tabel 4 of Dette, Podolskij and Vetter (2005). We observe that in most cases the new Kolmogorov-Smirnov test yields a substantial improvement with respect to power. For the sample size $n=100$ the procedure is more powerful for detecting the alternatives $\sigma^{2}(t, x)=1 ; 1+x^{2}$ and less powerful for the alternative $\sigma^{2}(t, x)=5|x|^{3 / 2}$. For the remaining two alternatives the new test yields slightly better results. One the other hand the asymptotic advantages of the Kolmogorov-Smirnov test become more visible for larger sample sizes $(n=200, n=500)$, where it outperforms the test based on the $L^{2}$-distance in all cases. For example, the power of the test of Dette, Podolskij and Vetter (2005) with $n=500$ observations is already achieved by the Kolmogorov-Smirnov test with $n=200$ observations. Except for the alternative $\sigma^{2}(t, x)=5|x|^{3 / 2}$ the power of the new test is substantially larger.

We finally note again that the power of the Kolmogorov-Smirnov test based on the process $\left(\hat{M}_{t}\right)_{t \in[0,1]}$ is even larger than the power obtained for $\left(\hat{N}_{t}\right)_{t \in[0,1]}$. Thus for high frequeny data the new tests are 
a substantial improvement of the currently available procedure for testing the parametric form of the diffusion coefficient in a stochastic differential equation.
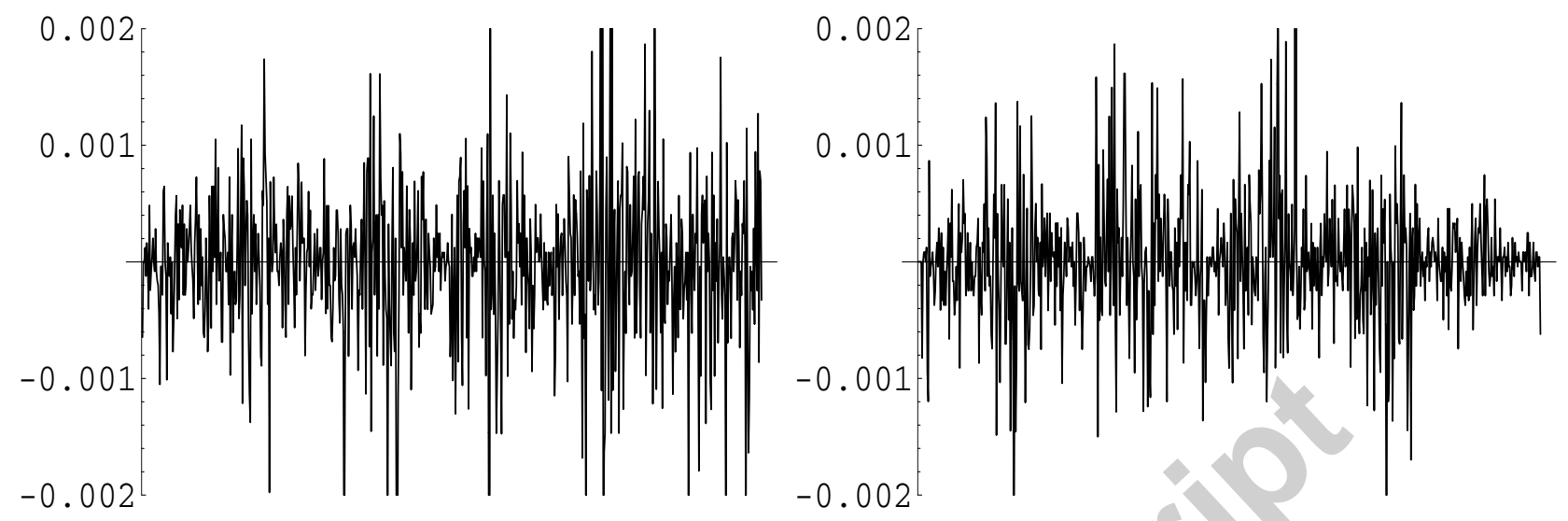

Figure 1 Log returns of the EUR/USD exchange rate for two different weeks.

4.3 Data Example. In this paragraph we apply the test based on the process $\left(N_{t}\right)_{t \in[0,1]}$ to tickby-tick data. As a specific example we consider the log returns of the excange rate between the EUR and the US dollar in 2004. The data were available for 10 weeks between February and April 2004 and approximately $710 \log$ returns were recorded per week. A typical picture for the 4th and 8th week is depicted in Figure 1.

We applied the proposed procedures to test the hypotheses $\bar{H}_{0}: \sigma^{2}(t, x)=\bar{\theta}_{1}, \bar{H}_{0}: \sigma^{2}(t, x)=\bar{\theta}_{1}|x|$, $\bar{H}_{0}: \sigma^{2}(t, x)=\bar{\theta}_{1} x^{2}$ and $\bar{H}_{0}: \sigma^{2}(t, x)=\bar{\theta}_{1}+\bar{\theta}_{2} x^{2}$. The corresponding $p$-values are depicted in Table 5. The null hypothesis $\bar{H}_{0}: \sigma^{2}(t, x)=\bar{\theta}_{1}$ is cleary rejected in all cases. For the hypotheses $\bar{H}_{0}: \sigma^{2}(t, x)=\bar{\theta}_{1}|x|$ and $\bar{H}_{0}: \sigma^{2}(t, x)=\bar{\theta}_{1} x^{2}$ the results do not indicate a clear structure. In the remaining case $\bar{H}_{0}: \sigma^{2}(t, x)=\bar{\theta}_{1}+\bar{\theta}_{2} x^{2}$ we observe relatively large $p$-values, which gives some evidence for the null hypothesis in all weeks under consideration. Note that some of these models have been used for describing interest rates (see e.g. Cox, Ingersoll and Ross, 1980, or Chan, Karolyi, Longstaff and Sanders, 1992). Our test indicates that a volatility of the form $\sigma^{2}(t, x)=\bar{\theta}_{1}+\bar{\theta}_{2} x^{2}$ is also appropriate to describe the variation in EUR/USD stock exchange data. 
Insert Table 5

\section{Appendix: Proofs}

Proof of Lemma 3.1. The proof of the following result is obtained along the lines of Dette, Podolskij and Vetter (2005) and therefore omitted.

Proof of Lemma 3.2. For the sake of brevity we restrict ourselves to a proof of asymptotic normality of the component

$$
\sqrt{n}\left(\hat{C}_{1}-C_{1}\right)
$$

The general case is shown by exactly the same arguments using the results of Barndorff-Nielsen, Graversen, Jacod, Podolskij and Shephard (2004). For a proof of the stable convergence of the statistic (39) we introduce the notation $\Delta_{i}^{n} X=X_{\frac{i}{n}}-X_{\frac{i-1}{n}}, \beta_{i}^{n}=\sqrt{n} \sigma\left(\frac{i-1}{n}, X_{\frac{i-1}{n}}\right) \Delta_{i}^{n} W, g(x)=|x|$, $\rho_{x}(f)=E[f(X)]$, where $X \sim N\left(0, x^{2}\right), \rho_{\sigma_{s}}(f)=\rho_{\sigma\left(s, X_{s}\right)}(f)$ and decompose the proof in three parts.

(1) We prove the assertion

$$
U^{n}=\frac{1}{\sqrt{n}} \sum_{i=1}^{n} \sigma_{1}\left(\frac{i-1}{n}, X_{\frac{i-1}{n}}\right)\left[g\left(\beta_{i}^{n}\right)-\rho_{\frac{\sigma_{-i-1}}{n}}(g)\right] \stackrel{\mathcal{D}_{s t}}{\longrightarrow} \nu \int_{0}^{1} \sigma_{1}\left(s, X_{s}\right) \sigma\left(s, X_{s}\right) d W_{s}^{\prime},
$$

where $\nu=\sqrt{\mu_{2}-\mu_{1}^{2}}$.

(2) We show the estimate $U^{n}-V^{n} \stackrel{P}{\longrightarrow} 0$, where the random variable $V_{n}$ is defined by

$$
V^{n}=\frac{1}{\sqrt{n}} \sum_{i=1}^{n} \sigma_{1}\left(\frac{i-1}{n}, X_{\frac{i-1}{n}}\right)\left[g\left(\sqrt{n} \Delta_{i}^{n} X\right)-E\left[g\left(\sqrt{n} \Delta_{i}^{n} X\right) \mid \mathcal{F}_{\frac{i-1}{n}}\right]\right]
$$

(3) We prove the convergence $\sqrt{n} \mu_{1}\left(\hat{C}_{1}-C_{1}\right)-V^{n} \stackrel{P}{\longrightarrow} 0$

Recalling the definition of $\hat{C}_{i}$ in (16) and observing (39), (1) - (3) it follows

$$
\sqrt{n}\left(\hat{C}_{1}-C_{1}\right) \stackrel{\mathcal{D}_{s t}}{\longrightarrow} \mu_{1}^{-1} \sqrt{\mu_{2}-\mu_{1}^{2}} \int_{0}^{1} \sigma_{1}\left(s, X_{s}\right) \sigma\left(s, X_{s}\right) d W_{s}
$$


which proves the assertion of Lemma 3.2 for the second component.

Proof of (1). We introduce the random variable $\xi_{i}^{n}=\frac{1}{\sqrt{n}} \sigma_{1}\left(\frac{i-1}{n}, X_{\frac{i-1}{n}}\right)\left[g\left(\beta_{i}^{n}\right)-\rho_{\sigma_{\frac{i-1}{n}}}(g)\right]$ and obtain the representation $U^{n}=\sum_{i=1}^{n} \xi_{i}^{n}$. Note that $g$ is an even function and observe the identities

$$
\begin{aligned}
E\left[\xi_{i}^{n} \mid \mathcal{F}_{\frac{i-1}{n}}\right] & =0, \quad E\left[\xi_{i}^{n} \Delta_{i}^{n} W \mid \mathcal{F}_{\frac{i-1}{n}}\right]=0 \\
E\left[\left|\xi_{i}^{n}\right|^{2} \mid \mathcal{F}_{\frac{i-1}{n}}\right] & =n^{-1}\left(\mu_{2}-\mu_{1}^{2}\right) \sigma_{1}^{2}\left(\frac{i-1}{n}, X_{\frac{i-1}{n}}\right) \sigma^{2}\left(\frac{i-1}{n}, X_{\frac{i-1}{n}}\right)
\end{aligned}
$$

Next, let $N$ be any bounded martingale on $\left(\Omega, \mathcal{F},\left(\mathcal{F}_{t}\right)_{0 \leq t \leq 1}, P\right)$, which is orthogonal to $W$ (this means that the quadratic variation process $\langle M, N\rangle_{t}$ is equal to 0$)$. It follows from BarndorffNielsen et al. (2004) $E\left[\xi_{i}^{n} \Delta_{i}^{n} N \mid \mathcal{F}_{\frac{i-1}{n}}\right]=0$, and finally Theorem IX 7.28 in Jacod and Shiryaev (2003) implies (40).

Proof of (2). We consider the representation $V_{n}-U_{n}=\sum_{i=1}^{n}\left(\zeta_{i}^{n}-E\left[\zeta_{i}^{n} \mid \mathcal{F}_{\frac{i-1}{n}}\right]\right)$, where the random variables $\zeta_{i}^{n}$ are given by

$$
\zeta_{i}^{n}=\frac{1}{\sqrt{n}} \sigma_{1}\left(\frac{i-1}{n}, X_{\frac{i-1}{n}}\right)\left[g\left(\sqrt{n} \Delta_{i}^{n} X\right)-g\left(\beta_{i}^{n}\right)\right]
$$

and note that it is sufficient to prove $\sum_{i=1}^{n} E\left[\left|\zeta_{i}^{n}\right|^{2}\right] \rightarrow 0$. For this we calculate using Burkholder inequality, Lemma 6.2 in Dette and Podolskij (2005) and (6)

$$
\begin{aligned}
\sum_{i=1}^{n} E\left[\left|\zeta_{i}^{n}\right|^{2}\right] & =\frac{1}{n} \sum_{i=1}^{n} E \sigma_{1}^{2}\left(\frac{i-1}{n}, X_{\frac{i-1}{n}}\right)\left[g\left(\sqrt{n} \Delta_{i}^{n} X\right)-g\left(\beta_{i}^{n}\right)\right]^{2} \\
& \leq \sum_{i=1}^{n} E \sigma_{1}^{2}\left(\frac{i-1}{n}, X_{\frac{i-1}{n}}\right)\left|\int_{\frac{i-1}{n}}^{\frac{i}{n}} b\left(s, X_{s}\right) d s+\int_{\frac{i-1}{n}}^{\frac{i}{n}} \sigma\left(s, X_{s}\right)-\sigma\left(\frac{i-1}{n}, X_{\frac{i-1}{n}}\right) d W_{s}\right|^{2} \\
& \leq 2 \sum_{i=1}^{n} E\left\{\sigma_{1}^{4}\left(\frac{i-1}{n}, X_{\frac{i-1}{n}}\right)\right\}^{\frac{1}{2}} E\left\{\left|\int_{\frac{i-1}{n}}^{\frac{i}{n}} b\left(s, X_{s}\right) d s\right|^{4}\right\}^{\frac{1}{2}} \\
& +E\left\{\sigma_{1}^{4}\left(\frac{i-1}{n}, X_{\frac{i-1}{n}}\right)\right\}^{\frac{1}{2}} E\left\{\left|\int_{\frac{i-1}{n}}^{\frac{i}{n}} \sigma\left(s, X_{s}\right)-\sigma\left(\frac{i-1}{n}, X_{\frac{i-1}{n}}\right) d W_{s}\right|^{4}\right\}^{\frac{1}{2}} \\
& =o(1),
\end{aligned}
$$

which completes the proof of (2). 
Proof of (3). Obviously, the assertion (3) follows from the statements

$$
\begin{gathered}
\frac{1}{\sqrt{n}} \sum_{i=1}^{n} \sigma_{1}\left(\frac{i-1}{n}, X_{\frac{i-1}{n}}\right) E\left[g\left(\sqrt{n} \Delta_{i}^{n} X\right)-g\left(\beta_{i}^{n}\right) \mid \mathcal{F}_{\frac{i-1}{n}}\right] \stackrel{P}{\longrightarrow} 0 \\
\sqrt{n} \sum_{i=1}^{n} \int_{\frac{i-1}{n}}^{\frac{i}{n}} \sigma_{1}\left(s, X_{s}\right) \rho_{\sigma_{s}}(g)-\sigma_{1}\left(\frac{i-1}{n}, X_{\frac{i-1}{n}}\right) \rho_{\frac{i-1}{n}}(g) d s \stackrel{P}{\longrightarrow} 0
\end{gathered}
$$

Note that $\sigma, \sigma_{1}>0$, which shows that $\rho_{\sigma_{s}}(g)=\mu_{1} \sigma_{s}$ and (46) follows along the lines of Dette, Podolskij and Vetter (2005). For a proof of (45) we define the set $A_{i}^{n}:=\left\{\left|\sqrt{n} \Delta_{i}^{n} X-\beta_{i}^{n}\right|>\left|\beta_{i}^{n}\right|\right\}$, and obtain the decomposition

$$
g\left(\sqrt{n} \Delta_{i}^{n} X\right)-g\left(\beta_{i}^{n}\right)=R_{i n}^{1}+R_{i n}^{2}-R_{i n}^{3}
$$

with $R_{\text {in }}^{1}=g^{\prime}\left(\beta_{i}^{n}\right)\left(\sqrt{n} \Delta_{i}^{n} X-\beta_{i}^{n}\right), R_{\text {in }}^{2}=\left[g\left(\sqrt{n} \Delta_{i}^{n} X\right)-g\left(\beta_{i}^{n}\right)\right] 1_{A_{i}^{n}}, R_{i n}^{3}=g^{\prime}\left(\beta_{i}^{n}\right)\left(\sqrt{n} \Delta_{i}^{n} X-\beta_{i}^{n}\right) 1_{A_{i}^{n}}$, where $1_{A}$ denotes the indicator function of the set $A$. Note that the decomposition (47) follows from the fact that the random variables $\sqrt{n} \Delta_{i}^{n} X$ and $\beta_{i}^{n}$ have the same sign if $\left(\sqrt{n} \Delta_{i}^{n} X, \beta_{i}^{n}\right)$ is an element of $\left(A_{i}^{n}\right)^{c}$ (here $B^{c}$ denotes the complement of the set $B$ ). Note also that $g^{\prime}$ is defined on $\mathbb{R} \backslash\{0\}$ and that $\sigma>0$. We now decompose $R_{\text {in }}^{1}$ as follows $R_{\text {in }}^{1}=R_{\text {in }}^{1.1}+R_{\text {in }}^{1.2}$, where

$$
\begin{aligned}
R_{i n}^{1.1} & :=\sqrt{n} g^{\prime}\left(\beta_{i}^{n}\right)\left[\frac{1}{n} b\left(\frac{i-1}{n}, X_{\frac{i-1}{n}}\right)+\int_{\frac{i-1}{n}}^{\frac{i}{n}} \sigma^{\prime}\left(\frac{i-1}{n}, X_{\frac{i-1}{n}}\right)\left(\int_{\frac{i-1}{n}}^{s} \sigma\left(\frac{i-1}{n}, X_{\frac{i-1}{n}}\right) d W_{t}\right) d W_{s}\right] \\
R_{i n}^{1.2} & :=\sqrt{n} g^{\prime}\left(\beta_{i}^{n}\right)\left[\int_{\frac{i-1}{n}}^{\frac{i}{n}} b\left(s, X_{s}\right)-b\left(\frac{i-1}{n}, X_{\frac{i-1}{n}}\right) d s+\int_{\frac{i-1}{n}}^{\frac{i}{n}} \sigma\left(s, X_{s}\right)-\sigma\left(\frac{i-1}{n}, X_{s}\right) d W_{s}\right. \\
& +\int_{\frac{i-1}{n}}^{\frac{i}{n}} \sigma^{\prime}\left(\frac{i-1}{n}, X_{\frac{i-1}{n}}\right)\left(\int_{\frac{i-1}{n}}^{s} b\left(t, X_{t}\right) d t+\int_{\frac{i-1}{n}}^{s} \sigma\left(t, X_{t}\right)-\sigma\left(\frac{i-1}{n}, X_{\frac{i-1}{n}}\right) d W_{t}\right) d W_{s} \\
& \left.+\frac{1}{2} \int_{\frac{i-1}{n}}^{\frac{i}{n}} \sigma^{\prime \prime}\left(\frac{i-1}{n}, \xi_{i}^{n}\right)\left(X_{s}-X_{\frac{i-1}{n}}\right)^{2} d W_{s}\right]=R_{i n}^{1.2 .1}+R_{i n}^{1.2 .2}+R_{i n}^{1.2 .3}+R_{i n}^{1.2 .4},
\end{aligned}
$$

the last line defines the random variables $R_{i n}^{1.2 . j}(j=1,2,3,4)$ and $\xi_{i}^{n}=\vartheta_{i}^{n} X_{\frac{i-1}{n}}+\left(1-\vartheta_{i}^{n}\right) X_{s}$ for some $\vartheta_{i}^{n} \in[0,1]$. Here $\sigma^{\prime}, \sigma^{\prime \prime}$ denote the first and the second derivative with respect to the second variable, respectively. Because $R_{i n}^{1.1}$ is an odd function of $\Delta_{i}^{n} W$ and $\Delta_{i}^{n} W$ is independent of the $\sigma$-field $\mathcal{F}_{\frac{i-1}{n}}$ we obtain $E\left[R_{i n}^{1.1} \mid \mathcal{F}_{\frac{i-1}{n}}\right]=0$. The Cauchy-Schwartz inequality, Burkholder inequality, Lemma 6.2 in Dette and Podolskij (2005) and (6) now yield $\sigma_{1}\left(\frac{i-1}{n}, X_{\frac{i-1}{n}}\right) E\left[R_{i n}^{1.2 . \ell} \mid \mathcal{F}_{\frac{i-1}{n}}\right]=O_{p}\left(n^{-1}\right)$, 
$\ell=1,2,3,4$ (uniformly with respect to $i=1, \ldots, n$ ), and we obtain

$$
\frac{1}{\sqrt{n}} \sum_{i=1}^{n} \sigma_{1}\left(\frac{i-1}{n}, X_{\frac{i-1}{n}}\right) E\left[R_{i n}^{1} \mid \mathcal{F}_{\frac{i-1}{n}}\right] \stackrel{P}{\longrightarrow} 0 .
$$

In order to derive similar estimates for $R_{i n}^{2}$ and $R_{i n}^{3}$ we note that it follows from Barndorff-Nielsen et al. (2004) that we may assume the existence of constants $c_{1}, c_{2}>0$ such that

$$
c_{1}<|\sigma|<c_{2}, \quad c_{1}<\left|\sigma_{j}\right|<c_{2}
$$

Observing the estimate $1_{A_{i}^{n}} \leq 1_{\left\{\left|\sqrt{n} \Delta_{i}^{n} X-\beta_{i}^{n}\right| \geq \varepsilon\right\}}+1_{\left\{\left|\beta_{i}^{n}\right|<\varepsilon\right\}}$, we obtain by using Burkholder inequality, Lemma in Dette and Podolskij (2005) and (6)

$$
E\left[1_{A_{i}^{n}} \mid \mathcal{F}_{\frac{i-1}{n}}\right] \leq \frac{E\left[\left|\sqrt{n} \Delta_{i}^{n} X-\beta_{i}^{n}\right|^{2}\right]}{\varepsilon^{2}}+K_{1} \varepsilon \leq K_{2}\left(\frac{1}{n \varepsilon^{2}}+\varepsilon\right)
$$

for some constants $K_{1}, K_{2}>0$. With the choice $\varepsilon=n^{-\frac{1}{3}}$ it therefore follows $E\left[1_{A_{i}^{n}} \mid \mathcal{F}_{\frac{i-1}{n}}\right]=O_{p}\left(n^{-\frac{1}{3}}\right)$. A further application of the Cauchy-Schwartz inequality yields $\sigma_{1}\left(\frac{i-1}{n}, X_{\frac{i-1}{n}}\right) E\left[R_{i n}^{\ell} \mid \mathcal{F}_{\frac{i-1}{n}}\right]=O_{p}\left(n^{-\frac{2}{3}}\right)$, $\ell=2,3$, which implies $1 / \sqrt{n} \sum_{i=1}^{n} \sigma_{1}\left(\frac{i-1}{n}, X_{\frac{i-1}{n}}\right) E\left[R_{i n}^{\ell} \mid \mathcal{F}_{\frac{i-1}{n}}\right] \stackrel{P}{\longrightarrow} 0, \ell=2,3$. The assertion (45) finally follows from (47), (48), which proves (3).

Proof of Theorem 3.3. Recall the definition of $\hat{B}_{t}^{i}(i=0, \ldots, d)$ in $(19)$, then the stable convergence (23) in Lemma 3.2 holds. Now an application of the Delta-method for stable convergence (see the proof of Theorem 4 in Dette, Podolskij and Vetter, 2005) yields weak convergence of the finite dimensional distributions, that is

$$
\sqrt{n}\left(\hat{M}_{t_{1}}-M_{t_{1}}, \ldots, \hat{M}_{t_{k}}-M_{t_{k}}\right)^{T} \stackrel{\mathcal{D}_{s t}}{\longrightarrow} \mu_{1}^{-1} \sqrt{\mu_{2}-\mu_{1}^{2}} V \int_{0}^{1} \Sigma_{t_{1}, \ldots t_{k}}^{\frac{1}{2}}\left(s, X_{s}\right) d W_{s}^{\prime}
$$

where the $k \times(d+k)$ matrix $V$ is defined in Theorem 3.3. We finally prove tightness of the sequence $\sqrt{n}\left(\hat{M}_{t}-M_{t}\right)$. For this we use the decomposition

$$
\sqrt{n}\left(\hat{M}_{t}-M_{t}\right)=\sqrt{n}\left(\hat{B}_{t}^{0}-B_{t}^{0}\right)+\sqrt{n} B_{t}^{T} D^{-1}(\hat{C}-C)+o_{p}(1)
$$

which follows from the definition of the processes $M_{t}$ and $\hat{M}_{t}$ in (13) and (18), respectively, and from Lemma 3.1. Tightness of the process $\sqrt{n}\left(\hat{B}_{t}^{0}-B_{t}^{0}\right)$ follows from Barndorff-Nielsen et al. (2004). 
For the second term in (52) we note that in view of (49) it is sufficient to prove

$$
\lim _{\delta \rightarrow 0} \limsup _{n \rightarrow \infty} P\left(\sqrt{n} \delta\left|D_{k l}^{-1}\right|\left|\hat{C}_{j}-C_{j}\right|>\varepsilon\right)=0
$$

for all $\varepsilon>0$ and all $1 \leq k, l, j \leq d$ where $D_{k l}^{-1}$ denote the element of the matrix $D^{-1}$ in the position $(k, \ell)$. An application of Cramer's rule, $(49)$ and Markov's inequality implies that

$$
\begin{aligned}
P\left(\sqrt{n} \delta\left|D_{k l}^{-1}\right|\left|\hat{C}_{j}-C_{j}\right|>\varepsilon\right) & =P\left(\sqrt{n} \delta\left|D_{k l}^{-1}\right|\left|\hat{C}_{j}-C_{j}\right|>\varepsilon, 0<\operatorname{det}(D)<\eta\right) \\
& +P\left(\sqrt{n} \delta\left|D_{k l}^{-1}\right|\left|\hat{C}_{j}-C_{j}\right|>\varepsilon, \operatorname{det}(D)>\eta\right) \\
& \leq P(0<\operatorname{det}(D)<\eta)+\frac{c n \delta^{2} E\left[\left|\hat{C}_{j}-C_{j}\right|^{2}\right]}{\eta \varepsilon^{2}}
\end{aligned}
$$

for some constant $c>0$ and for all $\eta>0$ (note that $\operatorname{det}(D)>0$, because $D$ is a positive definite matrix). Moreover, it follows from the proof of Lemma 3.2 that $E\left[n\left|\hat{C}_{j}-C_{j}\right|^{2}\right]=O(1)$. Now, for all $\varepsilon>0$, we choose $\eta$ small, then $\delta$ small. We immediately obtain (53) and by (52) the tightness of the process $\sqrt{n}\left(\hat{M}_{t}-M_{t}\right)$.

Proof of Corollary 3.4. Note that in the case $d=1$ and $\sigma_{1}=1$ we have for the quantities in (19) and (15) $\hat{B}_{t}^{1}=\lfloor n t\rfloor / n, \quad \hat{D}=1$, and the statistic $\hat{M}_{t}$ in (18) reduces to (29). Moreover, under the null hypothesis of homoscedasticity it follows in the case $k=2$ for the matrix $\tilde{V}$ in Theorem 3.3 $\tilde{V}=\left(-t_{1},-t_{2}\right)^{T}$. Note that under the hypothesis $H_{0}: \sigma\left(t, X_{t}\right)=\sigma$ we have $\theta^{\min }=\sigma$. The matrix $\Sigma_{t_{1}, t_{2}}$ in (24) can be calculated explicitly and does not depend on the process $X_{s}$. Consequently, the limiting process is Gaussian and determined by its covariance kernel. The representation

$$
V \int_{0}^{1} \Sigma_{t_{1}, t_{2}}\left(s, X_{s}\right) d s V^{T}=\sigma^{2}\left(\begin{array}{cc}
t_{1}\left(1-t_{1}\right) & t_{1} \wedge t_{2}-t_{1} t_{2} \\
t_{1} \wedge t_{2}-t_{1} t_{2} & t_{2}\left(1-t_{2}\right)
\end{array}\right)
$$

and Theorem 3.3 yield that under the null hypothesis of homoscedasticity the process $\sqrt{n} \hat{M}_{t}$ converges in law on $D[0,1]$, that is $\sqrt{n} \hat{M}_{t} \Longrightarrow \mu_{1}^{-1} \sqrt{\mu_{2}-\mu_{1}^{2}} \sigma B_{t}$, where $B_{t}$ denotes a Brownian bridge with covariance kernel $k\left(t_{1}, t_{2}\right)=t_{1} \wedge t_{2}-t_{1} t_{2}$. This completes the proof of Corollary 3.4. 
Proof of Theorem 3.6. We will only prove part (b) of the theorem. All other cases are treated by similar arguments. Since the drift function $b$ does not influence the limiting process we assume without loss of generality that $b=0$. With the notation $X_{t}^{H_{0}}=\sigma W_{t}$ we obtain the decomposition

$$
X_{t}=X_{t}^{H_{0}}+\gamma_{n} \int_{0}^{t} h\left(s, X_{s}\right) d W_{s}=X_{t}^{H_{0}}+\gamma_{n} X_{t}^{H_{1}}
$$

where the last identity defines the process $X_{t}^{H_{1}}$. This yields $\hat{N}_{t}=\sum_{k=1}^{\lfloor n t\rfloor}\left|\Delta_{i}^{n} X\right|^{2}-\frac{\lfloor n t\rfloor}{n} \sum_{k=1}^{n}\left|\Delta_{i}^{n} X\right|^{2}=$ $\hat{N}_{t}^{H_{0}}+S_{n}$. Here $\hat{N}_{t}^{H_{0}}$ denotes the process defined by (30), where the random variables $X_{\frac{k}{n}}$ have to be replaced by the corresponding quantities $X_{\frac{k}{n}}^{H_{0}}$ and $S_{n}=P_{n 1}+P_{n 2}-N_{n 1}-N_{n 2}$ with

$$
\begin{aligned}
& P_{n 1}=2 \gamma_{n} \sum_{k=1}^{\lfloor n t\rfloor} \Delta_{i}^{n} X^{H_{0}} \Delta_{i}^{n} X^{H_{1}}, \quad P_{n 2}=\gamma_{n}^{2} \sum_{k=1}^{\lfloor n t\rfloor}\left|\Delta_{i}^{n} X^{H_{1}}\right|^{2} \\
& N_{n 1}=2 \frac{\lfloor n t\rfloor}{n} \gamma_{n} \sum_{k=1}^{n} \Delta_{i}^{n} X^{H_{0}} \Delta_{i}^{n} X^{H_{1}}, \quad N_{n 2}=\frac{\lfloor n t\rfloor}{n} \gamma_{n}^{2} \sum_{k=1}^{n}\left|\Delta_{i}^{n} X^{H_{1}}\right|^{2}
\end{aligned}
$$

(here and in the following discussion the dependence of $P_{n j}$ and $N_{n j}(j=1,2)$ on the index $t$ will not be reflected by our notation). A straightforward calculation yields

$$
\begin{aligned}
& P_{n 1}=2 \gamma_{n} \sigma \int_{0}^{t} h\left(s, X_{s}\right) d s+o_{p}\left(\gamma_{n}\right), \quad P_{n 2}=\gamma_{n}^{2} \int_{0}^{t} h^{2}\left(s, X_{s}\right) d s+o_{p}\left(\gamma_{n}^{2}\right), \\
& N_{n 1}=2 t \gamma_{n} \sigma \int_{0}^{1} h\left(s, X_{s}\right) d s+o_{p}\left(\gamma_{n}\right), \quad N_{n 2}=t \gamma_{n}^{2} \int_{0}^{1} h^{2}\left(s, X_{s}\right) d s+o_{p}\left(\gamma_{n}^{2}\right),
\end{aligned}
$$

which gives $\sqrt{n} \hat{N}_{t}=\sqrt{n} \hat{N}_{t}^{H_{0}}+2 \sigma\left(\int_{0}^{t} h\left(s, X_{s}\right) d s-t \int_{0}^{1} h\left(s, X_{s}\right) d s\right)+o_{p}(1)$. The assertion now follows from Corollary 3.4.

Proof of Theorem 3.8. The convergence of finite dimensional distribution follows from Theorem 3.3 and the properties of stable convergence, so we only need to show the tightness of the sequence $\left(A_{n}(t) / \hat{s}_{t}\right)_{t \in[\underline{x}, \bar{x}]}$. In a first step we show that it is sufficient to prove tightness of the sequence $\left(A_{n}(t) / s_{t}\right)_{t \in[\underline{x}, \bar{x}]}$. For this we prove that

$$
\sup _{t \in[\underline{x}, \bar{x}]}\left|A_{n}(t)\left(\frac{1}{\hat{s}_{t}}-\frac{1}{s_{t}}\right)\right| \stackrel{P}{\longrightarrow} 0 .
$$

From Barndorff-Nielsen et al. (2004) we have $\sup _{t \in[\underline{x}, \bar{x}]}\left|A_{n}(t)\right|=O_{p}(1), \sup _{t \in[\underline{x}, \bar{x}]}\left|\hat{s}_{t}-s_{t}\right|=o_{p}(1)$. Observing (35) the inequality $s_{t}^{2} \geq \mu_{1}^{-2}\left(\mu_{2}-\mu_{1}^{2}\right) \lambda_{t}^{\min } \geq \mu_{1}^{-2}\left(\mu_{2}-\mu_{1}^{2}\right) \min _{t \in[\underline{x}, \bar{x}]} \lambda_{t}^{\min }>0$ holds for 
all $t \in[\underline{x}, \bar{x}]$, where $\lambda_{t}^{\text {min }}$ is the smallest eigenvalue of the positive definite matrix $\int_{0}^{1} \Sigma_{t}\left(s, X_{s}\right) d s$. For all $\varepsilon, \eta>0$ we have

$$
\begin{aligned}
P\left(\sup _{t \in[\underline{x}, \bar{x}]}\left|\frac{s_{t}-\hat{s}_{t}}{\hat{s}_{t} s_{t}}\right|>\varepsilon\right) & \leq P\left(0<\left(\mu_{1}^{-2}\left(\mu_{2}-\mu_{1}^{2}\right) \min _{t \in[\underline{x}, \bar{x}]} \lambda_{t}^{m i n}\right)^{\frac{1}{2}} \leq \eta\right)+P\left(\sup _{t \in[\underline{x}, \bar{x}]}\left|\hat{s}_{t}-s_{t}\right| \geq \frac{\eta}{2}\right) \\
& +P\left(\sup _{t \in[\underline{x}, \bar{x}]}\left|s_{t}-\hat{s}_{t}\right|>2 \eta^{2} \varepsilon\right) .
\end{aligned}
$$

Now, by choosing $\eta$ sufficiently small and then $n$ large we decuce that (55) holds. In a second step we show the tightness of the sequence $\left(A_{n}(t) / s_{t}\right)_{t \in[\underline{x}, \bar{x}]}$. For this it is sufficient to show

$$
\lim _{\delta \rightarrow 0} \limsup _{n \rightarrow \infty} P\left(\sup _{|t-h|<\delta}\left|\frac{A_{n}(t)}{s_{t}}-\frac{A_{n}(h)}{s_{h}}\right|>\varepsilon\right)=0
$$

for any $\varepsilon>0$. Observe that $A_{n}(t) / s_{t}-A_{n}(h) / s_{h}=\left(A_{n}(t)-A_{n}(h)\right) / s_{t}+A_{n}(t)\left(1 / s_{t}-1 / s_{h}\right)$. Since $s_{t}$ is continuous, we have for any $\varepsilon>0$

$$
\lim _{\delta \rightarrow 0} \limsup _{n \rightarrow \infty} P\left(\sup _{|t-h|<\delta}\left|A_{n}(t)\left(\frac{1}{s_{t}}-\frac{1}{s_{h}}\right)\right|>\varepsilon\right)=0,
$$

which can be shown by the same methods as (55). Moreover, we have for all $\eta>0$

$$
\begin{aligned}
\lim _{\delta \rightarrow 0} \limsup _{n \rightarrow \infty} P\left(\sup _{|t-h|<\delta}\left|\frac{A_{n}(t)-A_{n}(h)}{s_{t}}\right|>\varepsilon\right) & \leq P\left(0<\left(\mu_{1}^{-2}\left(\mu_{2}-\mu_{1}^{2}\right) \min _{t \in[\underline{x}, \bar{x}]} \lambda_{t}^{\min }\right)^{\frac{1}{2}} \leq \eta\right) \\
& +\lim _{\delta \rightarrow 0} \limsup _{n \rightarrow \infty} P\left(\sup _{|t-h|<\delta}\left|A_{n}(t)-A_{n}(h)\right|>\eta \varepsilon\right)
\end{aligned}
$$

From (53) we obtain

$$
\lim _{\delta \rightarrow 0} \limsup _{n \rightarrow \infty} P\left(\sup _{|t-h|<\delta}\left|\frac{A_{n}(t)-A_{n}(h)}{s_{t}}\right|>\varepsilon\right)=0
$$

by choosing $\eta$ small, which completes the proof of Theorem 3.8 .

Acknowledgements. The work of the authors was supported by the Deutsche Forschungsgmeinschaft (SFB 475, Komplexitätsreduktion in multivariaten Datenstrukturen). The authors are grateful to I. Gottschlich, who typed this paper with considerable technical expertise and to M. Vetter for computational assistance. We would also like to thank three referees for their constructive comments on an earlier version of this paper. 


\section{References}

Achieser, N.J., 1956, Theory of approximation, Dover Publications Inc., (New York).

Ait-Sahalia, Y., 1996, Testing continuous time models of the spot interest rate, Review of Financial Studies 9, 385-426.

Aldous, D.J. and G.K. Eagleson, 1978, On mixing and stability of limit theorems, Annals of Probability 6(2), 325-331.

Barndorff-Nielsen, O.E., S.E. Graversen, J. Jacod, M. Podolskij and N. Shephard, 2004, A central limit theorem for realised power and bipower variations of continuous semimartingales, Preprint, Ruhr-Universität Bochum. Available at:

http://www.ruhr-uni-bochum.de/mathematik3/preprint.htm

Black, F. and M. Scholes, 1973, The pricing of options and corporate liabilities, Journal of Political Economy 81, 637-654.

Brennan, M.J. and E.S. Schwartz, 1979, A continuous time approach to the pricing of bonds, Journal of Banking and Finance 3, 133-155.

Chan, K.C., A.G. Karolyi, F.A. Kongstaff and A.B. Sanders, 1992, An empirical comparison of alternative models of the short-term interest rate, Journal of Finance 47, 1209-1227.

Constantinides, G.M., 1992, A theory of the nominal term structure of interest rates, Review of Financial Studies 5, 531-552.

Corradi, V and H. White, 1999, Specification tests for the variance of a diffusion, Journal of Time Series Analysis 20, 253-270.

Courtadon, C., 1982, The pricing of options on default-free bonds, Journal of Financial and Quantitative Analysis 17, 75-100.

Cox, J.C., J.E. Ingersoll and S.A. Ross, 1985, A theory of the term structure of interest rates, Econometrica 53, 385-407. 
Cox, J.C., J.E. Ingersoll and S.A. Ross, 1980, An analysis of variable rate loan contracts, Journal of Finance 35, 389-403.

Dette, H. and C. von Lieres und Wilkau, 2003, On a test for a parametric form of volatility in continuous time financial models, Finance and Stochastics 7, 363-384.

Dette, H., M. Podolskij and M. Vetter, 2005, Estimation of integrated volatility in continuous time financial models with applications to goodness-of-fit testing, Scandinavian Journal of Statistics 33, 259-278.

Dette, H. and M. Podolskij, 2005, Testing the parametric form of the volatility in continuous time diffusion models - an empirical process approach, Technical report, Ruhr-Universität Bochum. Available at: http://www.ruhr-uni-bochum.de/mathematik3/preprint.htm

Duffie, J.D. and J.M. Harrison, 1993, Arbitrage pricing of Russian options and perpetual lookback options, Annals of Applied Probability 3, 641-651.

Jacod, J., 1997, On continuous conditional Gaussian martingales and stable convergence in law, Seminaire de Probabilites XXXI, 232-246.

Jacod, J. and A. N. Shiryaev, 2003, Limit theorems for stochastic processes, 2nd ed. Grundlehren der Mathematischen Wissenschaften, 288, Springer (Berlin).

Karatzas, I., 1988, On pricing of American options, Applied Mathematics and Optimization 17, $37-60$.

Karatzas, I. and S.E. Shreve, 1991, Brownian Motion and Stochastic Calculus, Springer (New York). Merton, R.C., 1973, Theory of rational option pricing, Bell Journal of Economics and Management Science 4, 141-183.

Merton, R.C., 1990, Continuous-Time Finance, Blackwell (Cambridge).

Renyi, A., 1963, On stable sequences of events, Sankhya-The Indian Journal of Statistics Series A 25, 293-302. 
Revuz, D. and M. Yor, 1999, Continuous Martingales and Brownian Motion, Springer (Berlin). Vasicek, O., 1977, An equilibrium characterization of the term structure, Journal of Financial Economics 5, 177-188. 


\begin{tabular}{|c|c|c|c|c|c|c|c|c|c|c|c|c|}
\hline \multicolumn{9}{|c|}{$M^{(n)}$} & \multicolumn{7}{c|}{$N^{(n)}$} \\
\hline$n$ & \multicolumn{2}{|c|}{100} & \multicolumn{2}{|c|}{200} & \multicolumn{2}{|c|}{500} & \multicolumn{2}{c|}{100} & \multicolumn{2}{c|}{200} & \multicolumn{2}{c|}{500} \\
\hline$b \backslash \alpha$ & $5 \%$ & $10 \%$ & $5 \%$ & $10 \%$ & $5 \%$ & $10 \%$ & $5 \%$ & $10 \%$ & $5 \%$ & $10 \%$ & $5 \%$ & $10 \%$ \\
\hline 0 & .044 & .098 & .045 & .099 & 0.046 & 0.093 & .047 & .082 & .041 & .077 & .041 & .098 \\
2 & .045 & .077 & .050 & .099 & 0.044 & 0.106 & .032 & .078 & .034 & .078 & .048 & .092 \\
$x$ & .045 & .095 & .044 & .081 & 0.042 & 0.091 & .033 & .089 & .033 & .081 & .041 & .082 \\
$2-x$ & .045 & .086 & .039 & .092 & 0.053 & 0.092 & .041 & .075 & .039 & .069 & .051 & .090 \\
$t x$ & .042 & .081 & .050 & .100 & 0.042 & 0.092 & .037 & .068 & .042 & .089 & .051 & .089 \\
\hline
\end{tabular}

Table 1 Approximation of the nominal level of the tests, which reject the null hypothesis of homoscedasticity for large values of the statistics $M^{(n)}$ and $N^{(n)}$. The critical values are obtained by the asymptotic law (36) and (37), respectively. 


\begin{tabular}{|c|c|c|c|c|c|c|c|c|c|c|c|c|}
\hline & \multicolumn{9}{|c|}{$M^{(n)}$} & \multicolumn{7}{c|}{$N^{(n)}$} \\
\hline$n$ & \multicolumn{2}{|c|}{100} & \multicolumn{2}{|c|}{200} & \multicolumn{2}{|c|}{500} & \multicolumn{2}{c|}{100} & \multicolumn{2}{c|}{200} & \multicolumn{2}{c|}{500} \\
\hline$\sigma / \alpha$ & $5 \%$ & $10 \%$ & $5 \%$ & $10 \%$ & $5 \%$ & $10 \%$ & $5 \%$ & $10 \%$ & $5 \%$ & $10 \%$ & $5 \%$ & $10 \%$ \\
\hline $1+x$ & .857 & .888 & .920 & .944 & .972 & .976 & .830 & .863 & .929 & .949 & .969 & .978 \\
$1+\sin (5 x)$ & .1 .000 & 1.000 & 1.000 & 1.000 & 1.000 & 1.000 & .998 & .999 & 1.000 & 1.000 & 1.000 & 1.000 \\
$1+x e^{t}$ & .972 & .981 & .990 & .993 & 0.999 & 0.999 & .947 & .968 & .989 & .996 & 0.998 & 0.998 \\
$1+x \sin (5 t)$ & .781 & .843 & .890 & .911 & .962 & .970 & .776 & .824 & .882 & .914 & .961 & .974 \\
$1+t x$ & .744 & .797 & .866 & .891 & .941 & .955 & .743 & .780 & .851 & .878 & .950 & .977 \\
\hline
\end{tabular}

Table 2 Rejection probabilities of the tests, which reject the null hypothesis of homoscedasticity for large values of thes tatistics $M^{(n)}$ and $N^{(n)}$. The critical values are obtained by the asymptotic law (36) and (37), respectively. 


\begin{tabular}{|c|c|c|c|c|c|c|c|c|c|c|c|c|}
\hline & \multicolumn{6}{|c|}{$\hat{M}_{t}$} & \multicolumn{5}{c|}{$\hat{N}_{t}$} \\
\hline$n$ & \multicolumn{2}{|c|}{100} & \multicolumn{2}{|c|}{200} & \multicolumn{2}{c|}{500} & \multicolumn{2}{c|}{100} & \multicolumn{2}{c|}{200} & \multicolumn{2}{c|}{500} \\
\hline$b / \alpha$ & $5 \%$ & $10 \%$ & $5 \%$ & $10 \%$ & $5 \%$ & $10 \%$ & $5 \%$ & $10 \%$ & $5 \%$ & $10 \%$ & $5 \%$ & $10 \%$ \\
\hline 0 & .125 & .195 & .081 & .135 & .074 & .133 & .052 & .110 & .050 & .099 & .061 & .114 \\
2 & .076 & .126 & .066 & .107 & .048 & .096 & .084 & .132 & .079 & .124 & .069 & .117 \\
$x$ & .094 & .148 & .071 & .128 & .048 & .100 & .069 & .129 & .057 & .117 & .054 & .100 \\
$2-x$ & .082 & .133 & .065 & .112 & .063 & .117 & .048 & .088 & .043 & .101 & .043 & .097 \\
$x t$ & .103 & .166 & .068 & .130 & .062 & .116 & .049 & .103 & .046 & .099 & .063 & .105 \\
\hline
\end{tabular}

Table 3 Simulated level of the bootstrap test for the hypothesis (39) based on the standardized Kolmogorov-Smirnov functional of the processes $\left(\hat{M}_{t}\right)_{t \in[0,1]}$ and $\left(\hat{N}_{t}\right)_{t \in[0,1]}$. 


\begin{tabular}{|c|c|c|c|c|c|c|c|c|c|c|c|c|}
\hline \multirow{3}{*}{$\begin{array}{c}n \\
\sigma^{2} / \alpha\end{array}$} & \multicolumn{6}{|c|}{$\hat{M}_{t}$} & \multicolumn{6}{|c|}{$\hat{N}_{t}$} \\
\hline & \multicolumn{2}{|c|}{100} & \multicolumn{2}{|c|}{200} & \multicolumn{2}{|c|}{500} & \multicolumn{2}{|c|}{100} & \multicolumn{2}{|c|}{200} & \multicolumn{2}{|c|}{500} \\
\hline & $5 \%$ & $10 \%$ & $5 \%$ & $10 \%$ & $5 \%$ & $10 \%$ & $5 \%$ & $10 \%$ & $5 \%$ & $10 \%$ & $5 \%$ & $10 \%$ \\
\hline $1+x^{2}$ & .516 & .587 & .652 & .720 & .831 & .885 & .352 & .467 & .502 & .627 & .752 & .828 \\
\hline 1 & .809 & .862 & .933 & .955 & .996 & .998 & .739 & .838 & .917 & .960 & .995 & .997 \\
\hline $5|x|^{3 / 2}$ & .371 & .516 & .511 & .638 & .743 & .838 & .252 & .310 & .388 & .534 & .485 & .598 \\
\hline $5|x|$ & .917 & .882 & .954 & .970 & .994 & .997 & .439 & .551 & .731 & .858 & .898 & .949 \\
\hline$(1+x)^{2}$ & .749 & .815 & .874 & .920 & .960 & .976 & .387 & .500 & .537 & .751 & .883 & .934 \\
\hline
\end{tabular}

Table 4 Simulated rejection probabilities of the bootstrap test for the hypothesis (39) based on the standardized Kolmogorov-Smirnov functional of the processes $\left(\hat{M}_{t}\right)_{t \in[0,1]}$ and $\left(\hat{N}_{t}\right)_{t \in[0,1]}$. 


\begin{tabular}{|l|c|c|c|c|c|c|c|c|c|c|}
\hline week & 1 th & 2 th & 3 th & 4 th & 5 th & 6 th & 7 th & 8 th & 9 th & 10 th \\
\hline$n$ & 714 & 714 & 713 & 714 & 714 & 714 & 708 & 714 & 718 & 710 \\
\hline$\sigma^{2}(t, x)=\theta_{1}$ & 0.000 & 0.026 & 0.000 & 0.002 & 0.002 & 0.000 & 0.004 & 0.000 & 0.001 & 0.010 \\
\hline$\sigma^{2}(t, x)=\theta_{1}|x|$ & 0.142 & 0.294 & 0.000 & 0.060 & 0.352 & 0.062 & 0.546 & 0.000 & 0.056 & 0.000 \\
\hline$\sigma^{2}(t, x)=\theta_{1} x^{2}$ & 0.748 & 0.714 & 0.000 & 0.976 & 0.774 & 0.368 & 0.634 & 0.000 & 0.710 & 0.000 \\
\hline$\sigma^{2}(t, x)=\theta_{1}+\theta_{2} x^{2}$ & 0.880 & 0.996 & 0.886 & 0.994 & 0.978 & 0.986 & 0.968 & 0.974 & 0.966 & 0.988 \\
\hline
\end{tabular}

Table 5 -values of the test based on the process $\left(N_{t}\right)_{t \in[0,1]}$ for various hypotheses on the volatility function. The table shows the results for ten weeks. The second row shows the number of the available data at each week. 